\title{
Documento de consenso para la detección y manejo de la enfermedad renal crónica
}

\author{
Alberto Martínez-Castelao', José L. Górriz¹, Jordi Bover ${ }^{1}$, Julián Segura-de la Morena², \\ Jesús Cebollada3 ${ }^{3}$ Javier Escalada4, Enric Esmatjes5 ${ }^{5}$ Lorenzo Fácila6, Javier Gamarra7, \\ Silvia Gràcia ${ }^{8}$, Julio Hernánd-Moreno7, José L. Llisterri-Caro ${ }^{9}$, Pilar Mazón ${ }^{6}$, Rosario Montañés ${ }^{8}$, \\ Francisco Morales-Olivas², Manuel Muñoz-Torres4, Pedro de Pablos-Velasco5 ${ }^{5}$, Ana de \\ Santiago ${ }^{9}$, Marta Sánchez-Celaya ${ }^{10}$, Carmen Suárez $^{3}$, Salvador Tranche $^{10}$ \\ ${ }^{1}$ Sociedad Española de Nefrología (S.E.N.) \\ ${ }^{2}$ Sociedad Española de Hipertensión Arterial-Liga Española para la Lucha contra la Hipertensión Arterial (SEH-LELHA) \\ ${ }^{3}$ Sociedad Española de Medicina Interna (SEMI) \\ ${ }^{4}$ Sociedad Española de Endocrinología y Nutrición (SEEN) \\ ${ }^{5}$ Sociedad Española de Diabetes (SED) \\ ${ }^{6}$ Sociedad Española de Cardiología (SEC) \\ ${ }^{7}$ Sociedad Española de Médicos Generalistas (AP) (SEMG) \\ ${ }^{8}$ Sociedad Española de Química Clínica (SEQC) \\ ${ }^{9}$ Sociedad Española de Medicina Rural y Generalista (AP) (SEMERGEN) \\ 10 Sociedad Española de Medicina de Familia y Comunitaria (AP) (SEMFyC)
}

Nefrologia 2014;34(2):243-62

doi:10.3265/Nefrologia.pre2014.Feb.12455

\section{RESUMEN}

La enfermedad renal crónica (ERC) es un importante problema de salud pública que puede afectar en sus diferentes estadios a cerca del $10 \%$ de la población española y que supone una elevada morbilidad y mortalidad, así como un importante consumo de recursos al Sistema Nacional de Salud. Diez sociedades científicas involucradas en el manejo del paciente renal nos hemos puesto de acuerdo para hacer una puesta al día del anterior documento de consenso sobre ERC de 2007. El presente es la edición abreviada del documento general extenso, que puede ser consultado en las páginas web de cada una de las sociedades firmantes. Contiene los siguientes aspectos: definición, epidemiología y factores de riesgo de la ERC. Criterios de diagnóstico, evaluación y estadiaje de la ERC, albuminuria y estimación del filtrado glomerular. Concepto y factores de progresión. Criterios de derivación a Nefrología. Seguimiento del paciente, actitudes y objetivos por especialidad. Prevención de la nefrotoxicidad. Detección del daño cardiovascular. Actitudes, estilo de vida y tratamiento: manejo de la hipertensión arterial, dislipemia, hiperglucemia, tabaquismo, obesidad, hiperuricemia, anemia, alteraciones del metabolismo mineral y óseo. Seguimiento coordinado por Atención Primaria-otras especialidades-Nefrología. Manejo del paciente en tratamiento renal sustitutivo, hemodiálisis, diálisis peritoneal y trasplante renal. Tratamiento paliativo de la uremia terminal. Esperamos que sirva de gran ayuda en el manejo multidisciplinar del paciente con ERC, a la vista de las recomendaciones más actualizadas.

Palabras clave: Enfermedad renal crónica. Consenso. Estadiaje de ERC. Detección de ERC. Albuminuria. Proteinuria. Filtrado glomerular. Cociente albúmina/creatinina en orina.

\section{Correspondencia: Alberto Martínez-Castelao}

Servicio de Nefrología. Hospital Universitario de Bellvitge.

Feixa Llarga sn. 08907 L'Hospitalet de Llobregat. Barcelona.

amartinez@bellvitgehospital.cat

amcastel@senefro.org
Consensus document for the detection and management of chronic kidney disease

\section{ABSTRACT}

Chronic kidney disease (CKD) is an important global health problem, involving to $10 \%$ of the Spanish population, promoting high morbidity and mortality for the patient and an elevate consumption of the total health resources for the National Health System. This is a summary of an executive consensus document of ten scientific societies involved in the care of the renal patient, that actualizes the consensus document published in 2007. The central extended document can be consulted in the web page of each society. The aspects included in the document are: Concept, epidemiology and risk factors for CKD. Diagnostic criteria, evaluation and stages of CKD, albuminuria and glomerular filtration rate estimation. Progression factors for renal damage. Patient remission criteria. Follow-up and objectives of each speciality control. Nephrotoxicity prevention. Cardiovascular damage detection. Diet, life-style and treatment attitudes: hypertension, dyslipidaemia, hyperglycemia, smoking, obesity, hyperuricemia, anemia, mineral and bone disorders. Multidisciplinary management for Primary Care, other specialities and Nephrology. Integrated management of CKD patient in haemodialysis, peritoneal dialysis and renal transplant patients. Management of the uremic patient in palliative care. We hope that this document may be of help for the multidisciplinary management of CKD patients by summarizing the most updated recommendations.

Keywords: Chronic kidney disease. Consensus document. Chronic kidney disease stages. Chronic kidney disease detection. Albuminuria. Proteinuria. Glomerular filtration rate. Urinary albumin/creatinin ratio.

\section{INTRODUCCIÓN}

La enfermedad renal crónica (ERC) es un importante problema de salud pública. Según los resultados del estudio 
EPIRCE (Epidemiología de la Insuficiencia Renal Crónica en España), diseñado para conocer la prevalencia de la ERC en España y promovido por la Sociedad Española de Nefrología (S.E.N.), con el apoyo del Ministerio de Sanidad y Consumo, el 9,24 \% de la población adulta sufre algún grado de $\mathrm{ERC}^{1}$. El 6,83\% de la población presenta una disminución del filtrado glomerular (FG) por debajo de $60 \mathrm{ml} /$ $\mathrm{min} / 1,73 \mathrm{~m}^{2}$, siendo este porcentaje del 20,6\% en mayores de 64 años. Además de la elevada prevalencia, la ERC se asocia a una importante morbimortalidad cardiovascular, así como a costes muy significativos. En España el coste anual asociado al tratamiento de las fases más avanzadas de la ERC se estima en más de 800 millones de euros anuales².

El hecho de plasmar en un documento tanto las estrategias de detección de la ERC como aquellas situaciones de riesgo con mayor probabilidad de progresar a enfermedad renal terminal o mayor morbimortalidad ayudará sin duda a detectar precozmente a aquellos individuos con mayor riesgo de progresión renal o de presentar complicaciones cardiovasculares. Igualmente, el establecimiento de estrategias de prevención y manejo de la ERC y sus complicaciones por parte de Atención Primaria, así como los criterios para la adecuada remisión de pacientes a Nefrología, completarán los aspectos abordados en este documento. Su finalidad es, pues, la prevención, detección, remisión al especialista y manejo de la ERC, con el fin de mejorar la salud renal y el pronóstico de nuestros pacientes.

Este documento de consenso surge de la necesidad de revisar y actualizar el anterior de S.E.N.-SEMFyC (Sociedad Española de Medicina de Familia y Comunitaria) de 2007 sobre $\mathrm{ERC}^{3}$, después de revisar exhaustivamente la literatura más reciente y las últimas recomendaciones de práctica clínica. La metodología empleada en él se ha basado en la revisión crítica de las principales guías clínicas sobre ERC y en el apoyo ocasional de los escasos estudios aleatorizados en pacientes con ERC.

Hemos creído oportuno implicar en su redacción a las sociedades científicas que incluyen entre sus objetivos el cuidado del enfermo renal y, por ello, surge el consenso entre las diez sociedades firmantes. Cada una de ellas ha nombrado a sus representantes (que figuran como autores) en la redacción del documento, que después ha sido sometido a la aprobación de sus respectivas juntas directivas. El documento ha estado expuesto en las páginas web de las diez sociedades, abierto a las posibles sugerencias de los miembros de cada una. Dichas sugerencias han sido incorporadas al texto íntegro definitivo y enviadas igualmente para ser expuestas en las respectivas páginas web de las sociedades firmantes.

El que ahora publicamos es un resumen más abreviado del documento original.

\section{ENFERMEDAD RENAL CRÓNICA: DEFINICIÓN Y EPIDEMIOLOGÍA}

Todas las guías consultadas ${ }^{4,5}$, incluidas las actuales guías KDIGO (Kidney Disease Improving Global Outcomes) 2012, publicadas en enero de $2013^{6}$, han confirmado la definición de ERC (independientemente del diagnóstico clínico) como la presencia durante al menos TRES MESES de:

- FGe (filtrado glomerular estimado) inferior a 60 $\mathrm{ml} / \mathrm{min} / 1,73 \mathrm{~m}^{2}$.

- O lesión renal.

La lesión renal se puede poner de manifiesto directamente a partir de alteraciones histológicas en la biopsia renal $\mathrm{o}$ indirectamente por la presencia de albuminuria, alteraciones en el sedimento urinario o a través de técnicas de imagen.

En España se estimó que el 9,24 \% de la población adulta padece algún grado de ERC, siendo el porcentaje sobre la población general del 6,83\% con ERC en estadios 3-5. La prevalencia de la ERC aumenta por el envejecimiento de la población, el incremento de la prevalencia de sus factores de riesgo, como la enfermedad cardiovascular, la diabetes mellitus (DM), la hipertensión arterial (HTA) o la obesidad y, obviamente, por su diagnóstico precoz. Se estima que la terapia sustitutiva renal consume el $2,5 \%$ del presupuesto del Sistema Nacional de Salud y más del $4 \%$ de Atención Especializada ${ }^{2}$.

\section{Factores de riesgo de la enfermedad renal crónica}

El modelo conceptual continuo de la $\mathrm{ERC}^{7}$ incluye factores de riesgo para cada una de sus fases, que se clasifican en factores de susceptibilidad, iniciadores, de progresión y de estadio final (tabla 1). Algunos factores de riesgo pueden ser a la vez de susceptibilidad, iniciadores y de progresión, como por ejemplo la HTA.

\section{Cribado de la enfermedad renal crónica}

El cribado de la ERC en poblaciones de riesgo debe hacerse mediante la evaluación del FGe y de la albuminuria al menos una vez al año. El diagnóstico no ha de basarse en una ÚNICA determinación de FGe y/o albuminuria y SIEMPRE debe confirmarse.

Recomendamos el cribado de la ERC en pacientes con HTA, DM tipo 2 o enfermedad cardiovascular establecida. Asimismo, se aconseja su cribado en personas mayores de 60 años, obesas (índice de masa corporal [IMC] $>35 \mathrm{~kg} / \mathrm{m}^{2}$ ), con DM tipo 1 con más de cinco años de evolución, familiares de primer grado de pacientes con enfermedad renal o con enfermedades renales hereditarias, enfermedades obs- 
Tabla 1. Factores de riesgo de la enfermedad renal crónica Factores de susceptibilidad: incrementan la posibilidad de daño renal

Edad avanzada

Historia familiar de ERC

Masa renal disminuida

Bajo peso al nacer

Raza negra y otras minorías étnicas

Hipertensión arterial

Diabetes

Obesidad

Nivel socioeconómico bajo

Factores iniciadores: inician directamente el daño renal

Enfermedades autoinmunes

Infecciones sistémicas

Infecciones urinarias

Litiasis renal

Obstrucción de las vías urinarias bajas

Fármacos nefrotóxicos, principalmente AINE

Hipertensión arterial

Diabetes

Factores de progresión: empeoran el daño renal y aceleran el deterioro funcional renal

Proteinuria persistente

Hipertensión arterial mal controlada

Diabetes mal controlada

Tabaquismo

Dislipemia

Anemia

Enfermedad cardiovascular asociada

Obesidad

\section{Factores de estadio final: incrementan la} morbimortalidad en situación de fallo renal

Dosis baja de diálisis $(\mathrm{Kt} / \mathrm{N})^{\mathrm{a}}$

Acceso vascular temporal para diálisis

Anemia

Hipoalbuminemia

Derivación tardía a Nefrología

AINE: antiinflamatorios no esteroideos; ERC: enfermedad renal crónica.

${ }^{a} \mathrm{Kt} / \mathrm{V}: \mathrm{K}=$ depuración de urea en el dializador; $\mathrm{t}$ = tiempo; $\mathrm{V}=$ volumen de distribución de la urea. La cifra resultante se utiliza para cuantificar la suficiencia de la dosis de diálisis.

tructivas del tracto urinario, pacientes en tratamiento prolongado con fármacos nefrotóxicos (incluidos antiinflamatorios no esteroideos [AINE]), sujetos con otros factores de riesgo de enfermedad cardiovascular (hiperlipidemia, síndrome metabólico, fumadores), antecedentes de insuficiencia renal aguda, así como aquellos con infecciones crónicas, enfermedades autoinmunes y neoplasias que estén asociadas a ERC.

\section{DIAGNÓSTICO DE LA ENFERMEDAD RENAL CRÓNICA}

\section{Estimación del filtrado glomerular}

La concentración de creatinina sérica no se debería utilizar como única prueba para evaluar la función renal, siendo el FG la mejor herramienta para hacerlo. El cálculo del FG a partir del aclaramiento de creatinina (medición de la concentración de creatinina en suero y orina de 24 horas) presenta una serie de inconvenientes, como son la sobreestimación del FG y la problemática que supone la recogida de orina de 24 horas tanto para el paciente como para los laboratorios. La medida del aclaramiento de creatinina mediante la recogida de orina de 24 horas no mejora, salvo en determinadas circunstancias, la estimación del FG a partir de ecuaciones.

Recomendamos la estimación del FG mediante ecuaciones obtenidas a partir de la medida de la concentración de creatinina sérica, la edad, el sexo y la etnia. Estas ecuaciones son más exactas que la medida de la creatinina sérica aislada. Las más utilizadas son las derivadas del estudio Modification of Diet in Renal Disease (MDRD-4 o MDRD-IDMS) ${ }^{8}$, en función de si el método usado por el laboratorio para la medida de la creatinina sérica presenta o no trazabilidad (tabla 2) frente al procedimiento de medida de referencia de espectrometría de masasdilución isotópica (IDMS), siendo recomendable este último.

La ecuación CKD-EPI (Chronic Kidney Disease-Epidemiology Collaboration $)^{9}$, usando también métodos de creatinina estandarizados, proporciona ventajas adicionales respecto al MDRD-IDMS, dado que presenta una mayor exactitud y mejora la capacidad predictiva del FG (especialmente entre valores de 60 y $90 \mathrm{ml} / \mathrm{min} / 1,73 \mathrm{~m}^{2}$ ), así como la predicción de mortalidad global y cardiovascular o del riesgo de presentar ERC terminal ${ }^{10}$. Por ello se considera que CKD-EPI debería sustituir las fórmulas anteriores (tabla 3). Las nuevas guías KDIGO 2012 consideran aceptable el uso de fórmulas alternativas si se ha mostrado que mejoran la exactitud en comparación con la fórmula de CKD-EPI ${ }^{6}$.

Esta ecuación ha mostrado su superioridad frente a otras de estimación del FG basadas en la concentración sérica de creatinina (MDRD), cistatina C o en la combinación de ambas ${ }^{10-13}$.

Tabla 2. Ecuaciones a utilizar para métodos de medida de creatinina sin trazabilidad a IDMS (no estandarizados)

$\mathrm{FGe}=186 \times(\text { creatinina })^{-1,154} \times(\text { edad })^{-0,203} \times 0,742($ si mujer $)$ $x$ 1,21 (si etnia negra)

FGe: filtrado glomerular estimado $\left(\mathrm{ml} / \mathrm{min} / 1,73 \mathrm{~m}^{2}\right)$.

Creatinina $=$ concentración sérica de creatinina en $\mathrm{mg} / \mathrm{dl}$. Edad en años. 
El uso de la ecuación de Cockcroft-Gault $(\mathrm{C}-\mathrm{G})^{14}$, aunque se ha utilizado clásicamente en el ajuste de dosis de fármacos y ha sido referencia para la valoración de estados de hiperfiltración, debería desaconsejarse. Dicha ecuación no ha sido reformulada para valores de creatinina obtenidos por procedimientos adecuados y no puede ser reexpresada para los métodos actuales de medida de creatinina, por lo que no debería usarse. Las ecuaciones CKD-EPI o MDRD-IDMS pueden ser utilizadas con este fin, ya que se basan en procedimientos de medida de creatinina estandarizados. El FG obtenido a partir de MDRD o CKD-EPI es útil en cuanto al ajuste de dosis de fármacos, pues correlaciona mejor que el obtenido por C-G para valores inferiores a $60 \mathrm{ml} / \mathrm{min} / 1,73 \mathrm{~m}^{2}$, que son los mayoritariamente susceptibles de necesidad de ajuste de dosis y están disponibles en los informes de los laboratorios clínicos, al contrario que C-G $\mathrm{G}^{10,11,15,16}$.

Tabla 3. Ecuaciones a utilizar para métodos de medida de creatinina con trazabilidad a IDMS (estandarizados)

\section{Ecuación CKD-EPI}

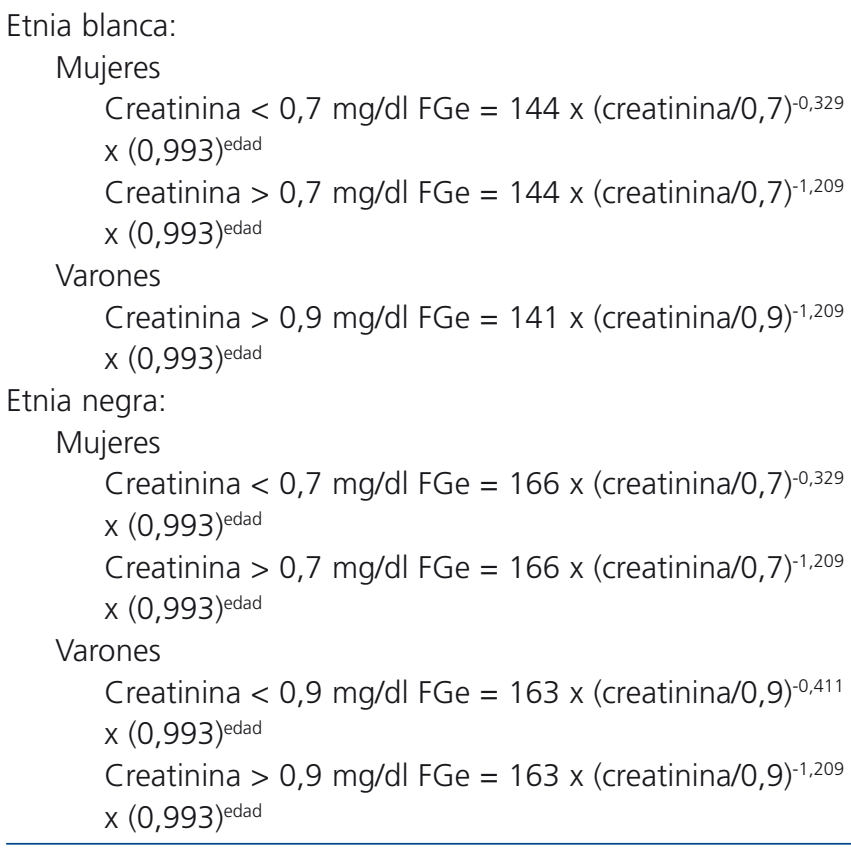

\section{Ecuación MDRD-IDMS}

$\mathrm{FGe}=175 \times(\text { creatinina })^{-1,154} \times(\text { edad })^{-0,203} \times 0,742$ (si mujer $) \times$ 1,21 (si etnia negra)

CKD-EPI: Chronic Kidney Disease-Epidemiology Collaboration; FGe: filtrado glomerular estimado $\left(\mathrm{ml} / \mathrm{min} / 1,73 \mathrm{~m}^{2}\right)$;

IDMS: espectrometría de masas-dilución isotópica;

MDRD: Modification of Diet in Renal Disease.

Creatinina = concentración sérica de creatinina en mg/dl. Edad en años.
Los valores obtenidos mediante las ecuaciones de MDRD o CKD-EPI están ajustados a superficie corporal (SC) para $1,73 \mathrm{~m}^{2}$. Pero en el caso de necesidad de utilizar la fórmula o de ajustar fármacos especialmente tóxicos o con escaso margen terapéutico en pacientes con desviaciones importantes de la SC, los valores del FG no se deberían estandarizar a $1,73 \mathrm{~m}^{2}$. En estos casos basta con multiplicar el resultado del laboratorio expresado en $\mathrm{ml} / \mathrm{min} / 1,73 \mathrm{~m}^{2}$ por el cociente de la $\mathrm{SC}$ real del paciente dividida por $1,73 \mathrm{~m}^{2}\left(\mathrm{FG} \times \mathrm{SC} / 1,73 \mathrm{~m}^{2}\right)$.

En general, el uso de las ecuaciones para la estimación del FG (MDRD y CKD-EPI) es inadecuado en una serie de situaciones clínicas, especialmente en personas con peso corporal extremo $\left(\mathrm{IMC}<19 \mathrm{~kg} / \mathrm{m}^{2}\right.$ o $>35 \mathrm{~kg} / \mathrm{m}^{2}$ ), dietas especiales o malnutrición, alteraciones de la masa muscular, amputaciones, $<18$ años, hepatópatas, embarazadas, fracaso renal agudo y en el estudio de potenciales donantes de riñón. En estos casos, para una adecuada medida de la función renal se requerirá la recogida de orina de 24 horas para el cálculo del aclaramiento de creatinina ${ }^{3}$.

Hasta ahora ninguna guía de práctica clínica ha incluido el uso de cistatina C o FG estimado a partir de ella como parámetro de cribado de la ERC, pero las nuevas guías KDIGO $2012^{6}$ sugieren la medida de cistatina $\mathrm{C}$ en adultos con FG entre 45 y $59 \mathrm{ml} / \mathrm{min} / 1,73 \mathrm{~m}^{2}$, sin otros marcadores de lesión renal, si se requiere una confirmación diagnóstica de ERC. Se debería usar entonces la ecuación CKD-EPI para cistatina C recientemente publicada ${ }^{6}$.

\section{Evaluación de la lesión renal}

\section{Albuminuria (excreción urinaria de albúmina)}

La albuminuria constituye, junto con el FG, la base del diagnóstico y estadiaje actual de la ERC. La presencia de concentraciones elevadas de proteína o albúmina en la orina, de forma persistente, no solo es un signo de lesión renal, sino muchas veces también de «daño sistémico», más allá del riñón. Distintos estudios han mostrado la importancia de la proteinuria en la patogenia de la progresión de la ERC, así como la relación de la albuminuria con el pronóstico renal y con la mortalidad en diversas poblaciones de modo independiente del FG y otros factores de riesgo clásicos de enfermedad cardiovascular.

Recomendamos prescindir del uso de términos como micro o macroalbuminuria y emplear el término de albuminuria o excreción urinaria de albúmina, y el valor absoluto del cociente albúmina/creatinina en orina (CAC), preferentemente en la primera orina de la mañana. El CAC es un marcador más sensible que la proteinuria en el contexto de ERC secundaria a DM, HTA o enfermedad glomerular, que son las causas más frecuentes de ERC en el adulto. 
En el caso de pacientes con ERC diagnosticada y proteinuria significativa (por ejemplo, CAC $>300-500 \mathrm{mg} / \mathrm{g}$ ), se podría realizar la monitorización a partir del cociente proteínas/creatinina en orina por tratarse de una determinación más económica y porque, a medida que se incrementa la proteinuria, especialmente en proteinuria nefrótica, el CAC es menos sensible. También se recomienda la utilización del cociente proteínas/creatinina en orina en pacientes con sospecha de patología intersticial y de nefrotoxicidad por antirretrovirales, ya que en ambas situaciones la proteinuria está constituida fundamentalmente por proteínas de bajo peso molecular, diferente a la albúmina. Para considerar que una persona tiene albuminuria, son necesarios dos valores elevados en tres muestras obtenidas durante un período de 3 a 6 meses.

El valor y la persistencia de la albuminuria se relacionan estrechamente con el pronóstico renal y vital de los pacientes con ERC, pero también debemos considerar que la albuminuria es un marcador importante e independiente de riesgo cardiovascular global (disfunción endotelial, remodelado arterial), y no únicamente de enfermedad renal. La presencia única de albuminuria, sin ninguna otra manifestación de daño renal, está puesta en entredicho por varios autores como un criterio único y específico de ERC, ya que se puede detectar en otras patologías (obesidad, tabaquismo, dermatitis, artritis).

Debe recordarse que la determinación de proteinuria incluye no solo la cuantificación de albúmina, sino también la de proteínas de bajo peso molecular, como proteínas de origen tubular o cadenas ligeras de inmunoglobulinas.

\section{Alteraciones en el sedimento urinario}

La presencia en el sedimento urinario de hematuria y/o leucocituria durante más de tres meses, una vez se ha descartado la causa urológica o la infección de orina (incluida la tuberculosis urinaria), puede ser también indicio de ERC.

\section{Imágenes radiológicas patológicas}

La ecografía renal permite, en primer lugar, descartar la presencia de patología obstructiva de la vía urinaria, pero también identificar anormalidades estructurales que indican la presencia de daño renal. Los quistes renales simples aislados NO son un criterio por ellos mismos de daño renal.

\section{Alteraciones histológicas}

La indicación de biopsia forma parte del ámbito del especialista en Nefrología.

\section{Nuevo estadiaje de la enfermedad renal crónica}

Recientemente, a partir de los resultados de distintos estudios clínicos que incluyen individuos normales, individuos con riesgo de desarrollar ERC y pacientes con ERC, la organización internacional $\mathrm{KDIGO}^{6}$ ha establecido una nueva clasificación pronóstica de la ERC basada en estadios de FGe y albuminuria. Esta clasificación contempla una división de seis categorías de riesgo en función del FGe (G1-G5), que se complementan con tres categorías de riesgo según la concentración del CAC: A1 para valores óptimos o normales-altos ( $<30 \mathrm{mg} / \mathrm{g}$ o $<3 \mathrm{mg} / \mathrm{mmol}$ ); A2 para valores moderadamente aumentados (30-299 mg/g o 3-29 mg/mmol); y A3 para valores muy aumentados ( $\geq 300 \mathrm{mg} / \mathrm{g} \mathrm{o} \geq 30 \mathrm{mg} / \mathrm{mmol}$ ), respectivamente (tabla 4).

\section{DEFINICIÓN DE PROGRESIÓN DE LA ENFERMEDAD RENAL CRÓNICA}

La tasa media de disminución anual del FG es muy variable, siendo mayor en pacientes con proteinuria importante, DM o HTA.

\section{Puntos clave}

1. Tasa de progresión renal normal: $0,7-1 \mathrm{ml} / \mathrm{min} / 1,73 \mathrm{~m}^{2}$ año a partir de los 40 años ${ }^{6}$.

2. Se puede considerar que un paciente presenta progresión renal: descenso del FG $>5 \mathrm{ml} / \mathrm{min} /$ año o $>10 \mathrm{ml} / \mathrm{min}$ en cinco años ${ }^{1}$.

3. Se deberá definir la progresión con base en dos vertientes:

- Progresión a una categoría superior o más grave de deterioro en la función renal (estadio 1-5) o de albuminuria $(<30,30-299,>300 \mathrm{mg} / \mathrm{g})$.

- Porcentaje de cambio respecto a la situación basal (> $25 \%$ de deterioro en el FG) o más del $50 \%$ de incremento en el cociente CAC.

4. Para la valoración de la progresión renal se recomienda la estimación del FG basal y la albuminuria, así como identificar aquellos factores de progresión renal. Ello indicará la frecuencia de determinación de sucesivos controles analíticos.

5. Para asegurar la exactitud de la medición de la tasa de deterioro renal, dicha guía aconseja realizar dos medidas del FGe en un período no inferior a tres meses y descartar una disminución debida a una insuficiencia renal aguda o al inicio de tratamiento con fármacos que afectan a la hemodinámica glomerular (inhibidores de la enzima convertidora de la angiotensina [IECA], antagonistas de los receptores de angiotensina II [ARA II], AINE, diuréticos).

6 En pacientes con un nuevo diagnóstico de ERC (por primera vez), se ha de repetir la estimación del FG en un período no inferior a tres meses para descartar deterioro renal agudo por factores exógenos (diarrea, vómitos, depleción por diuréticos, o cualquier fármaco que afecte la hemodinámica glomerular, como IECA, ARA II, inhibidores 


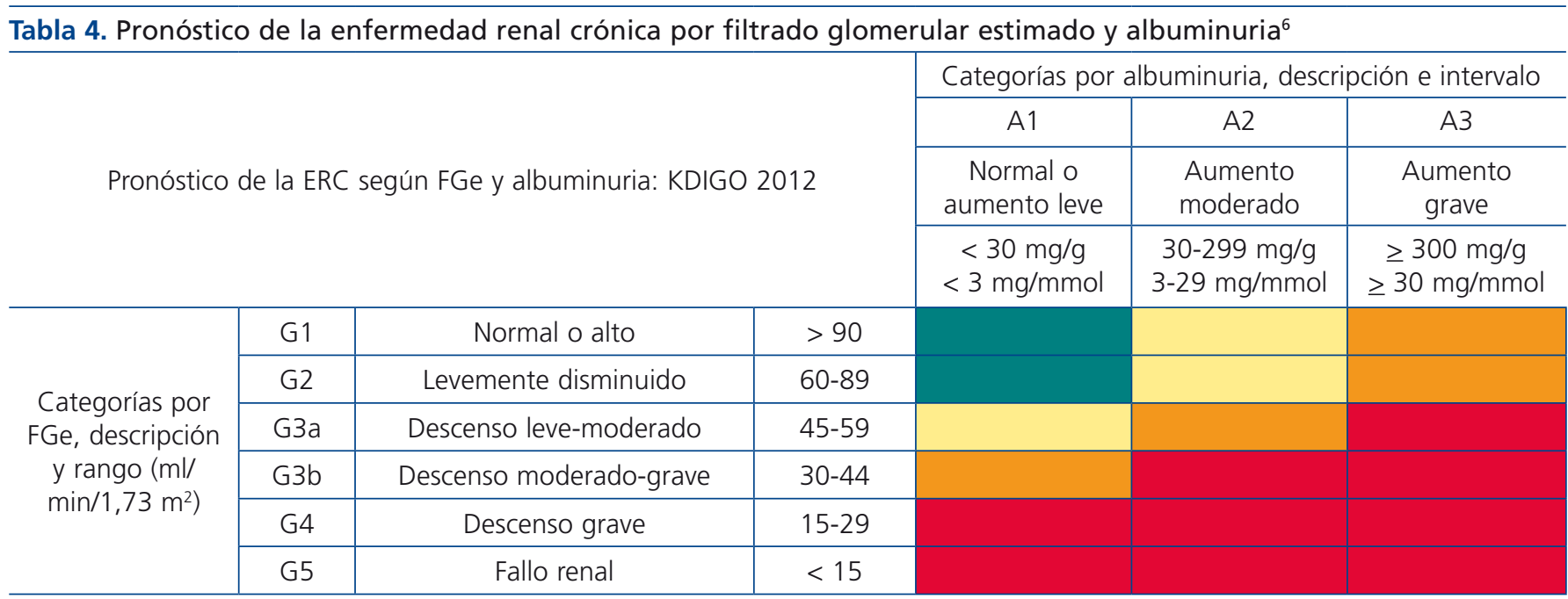

ERC: enfermedad renal crónica; FGe: filtrado glomerular estimado; KDIGO: Kidney Disease Improving Global Outcomes. Nota: Los colores mostrarían el riesgo relativo ajustado para cinco eventos (mortalidad global, mortalidad cardiovascular, fracaso renal tratado con diálisis o trasplante, fracaso renal agudo y progresión de la enfermedad renal) a partir de un metaanálisis de cohortes de población general. El riesgo menor corresponde al color verde (categoría «bajo riesgo»; si no hay datos de lesión renal, no se puede catalogar siquiera como ERC), seguido del color amarillo (riesgo «moderadamente aumentado»), naranja («alto riesgo») y rojo («muy alto riesgo»), que expresan riesgos crecientes para los eventos mencionados. Reproducido con permiso de $\mathrm{KDIGO}^{6}$.

Cociente albúmina/creatinina: $1 \mathrm{mg} / \mathrm{g}=0,113 \mathrm{mg} / \mathrm{mmol} .30 \mathrm{mg} / \mathrm{g}(3,4 \mathrm{mg} / \mathrm{mmol})$.

Como puede apreciarse, los estadios según el FG se denominan ahora G1 a G5 y se confirma la división del estadio 3 en dos subgrupos: G3a y G3b, división también útil para determinar la prioridad de la derivación y las diferencias de riesgo.

directos de la renina). Si la situación clínica lo indica, podría repetirse en un período inferior a tres meses. En pacientes con ERC conocida, se sugiere medir el FGe y el CAC anualmente si presentan bajo riesgo de progresión, y más frecuentemente si presentan riesgo elevado de progresión.

\section{PREDICTORES DE PROGRESIÓN}

Los factores predictores de progresión renal se muestran en la tabla $5^{5,17-28}$.

Tabla 5. Factores predictores de progresión de la enfermedad renal crónica

Factores $^{17-18}$
Proteinuria'19,20
Hipertensión arterial21,22
Diabetes mellitus ${ }^{23}$
Enfermedad cardiovascular $^{24}$
Tabaquismo $^{25}$
Obesidad
Raza negra o asiática $^{27}$
Tratamiento crónico con AINE $^{28}$
Obstrucción del tracto urinario

AINE: antiinflamatorios no esteroideos.

\section{CRITERIOS DE DERIVACIÓN A NEFROLOGÍA (figura 1)}

La derivación a Nefrología se hará teniendo en cuenta el estadio de ERC, la velocidad de progresión de la insuficiencia renal, el grado de albuminuria, la presencia de signos de alarma, la comorbilidad asociada y la situación funcional del paciente pa $^{3,5,29}$.

En líneas generales, se deberán remitir al especialista en Nefrología los pacientes con $\mathbf{F G e}<\mathbf{3 0} \mathbf{~ m l} / \mathbf{m i n} / \mathbf{1}, \mathbf{7 3} \mathbf{~ m}^{2}$ (excepto $>80$ años sin progresión renal, albuminuria $<300 \mathrm{mg} / \mathrm{g}$ ).

\section{Según filtrado glomerular:}

- Todos los pacientes con FGe $<30 \mathrm{ml} / \mathrm{min} / 1,73 \mathrm{~m}^{2}$, excepto los pacientes $>80$ años sin progresión renal.

- Pacientes $>80$ años y con FGe $<20 \mathrm{ml} / \mathrm{min} / 1,73 \mathrm{~m}^{2}$, si su situación general lo aconseja, se pueden remitir para una valoración nefrológica y pactar el tratamiento. Se recomienda que el paciente candidato se remita a Nefrología al menos un año antes del inicio de la terapia sustitutiva renal. Aunque este período no es fácil de calcular, la presencia de progresión renal (ver punto 5) puede servir de guía. El objetivo es evitar que un paciente candidato a terapia sustitutiva renal requiera diálisis no programada.

- Los pacientes < 70 años con FGe entre 30-45 ml/ min/1,73 $\mathrm{m}^{2}$ deberán realizarse una monitorización más frecuente (cada 3-6 meses) y remitirse a Nefrología solo 


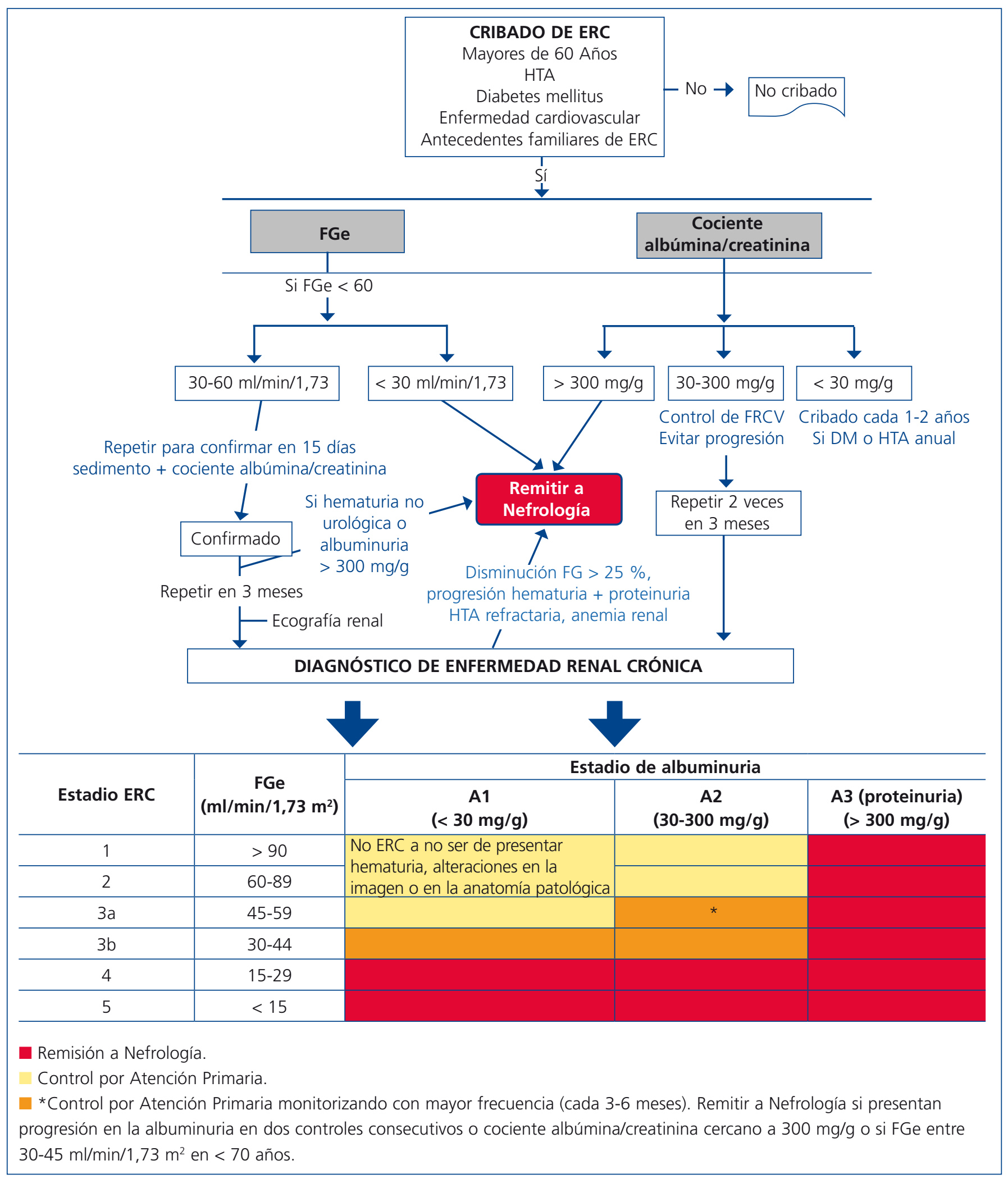

Figura 1. Algoritmo de derivación a Nefrología.

DM: diabetes mellitus; ERC: enfermedad renal crónica; FGe: filtrado glomerular estimado; FRCV: factores de riesgo cardiovascular; HTA: hipertensión arterial.

En $>\mathbf{8 0}$ años no remitir, incluso a pesar de $\mathrm{FGe}<30 \mathrm{ml} / \mathrm{min} / 1,73 \mathrm{~m}^{2}$, a no ser que presenten alguno de los siguientes signos de alarma: albuminuria $>300 \mathrm{mg} / \mathrm{g}$, hematuria no urológica, progresión renal ( $>5 \mathrm{ml} / \mathrm{min} / 1,73 \mathrm{~m}^{2} / \mathrm{año}$ ) o $\mathrm{FGe}>25 \%$ en un mes, $\mathrm{FGe}<20 \mathrm{ml} / \mathrm{min} / 1,73 \mathrm{~m}^{2}$ o previsión de necesidad de terapia sustitutiva renal. 
en caso de progresión de la albuminuria en dos controles consecutivos o cociente CAC cercano a $300 \mathrm{mg} / \mathrm{g}$.

Según albuminuria: cociente CAC $>300 \mathrm{mg} / \mathrm{g}$, equivalente a proteinuria $>300 \mathrm{mg} / 24$ horas.

\section{Otros motivos:}

- Deterioro agudo en la función renal (caída del FGe $>25 \%$ ) en menos de un mes, descartados factores exógenos (diarrea, vómitos, depleción por diuréticos en tratamiento con IECA o ARA II, o inhibidores directos de la renina).

- Pacientes que presenten progresión renal (> $5 \mathrm{ml} / \mathrm{min} /$ año) (ver definición arriba).

- ERC e HTA refractaria al tratamiento (> 140/90 mmHg) con tres fármacos a plena dosis, uno de ellos diurético.

- Alteraciones en el potasio ( $>5,5 \mathrm{mEq} / 1$ o $<3,5 \mathrm{mEq} / 1$ sin recibir diuréticos).

- Anemia: hemoglobina [Hb] < 10,5 g/dl con ERC a pesar de corregir ferropenia (índice de saturación de transferrina [IST] $>20 \%$ y ferritina $>100$ ).

- Presencia de signos de alarma:

- Hematuria no urológica asociada a proteinuria.

- Disminución del FGe > $25 \%$ en menos de un mes o un incremento de la creatinina plasmática $>25 \%$ en menos de un mes, descartados factores exógenos (diarrea, vómitos, depleción por diuréticos en tratamiento con IECA o ARA II, o inhibidores directos de la renina).

El seguimiento podrá ser en Atención Primaria o conjunto, según los casos.

\section{En el caso de pacientes ancianos ( $>80$ años)}

Dado que la progresión de la ERC en población anciana es muy poco frecuente, se puede aceptar que los pacientes mayores de 80 años con función renal estable o con lento deterioro de esta $(<5 \mathrm{ml} / \mathrm{min} /$ año $)$ sin proteinuria ni anemia o signos de alarma pueden llevar seguimiento con actitud conservadora en Atención Primaria ${ }^{30,31}$.

En el mismo sentido, los pacientes ancianos con ERC estadio 5 con expectativa de vida corta $(<6$ meses), mala situación funcional (dependencia de las actividades de la vida diaria, demencia, etc.), comorbilidad asociada grave o que no acepten diálisis podrán ser subsidiarios de tratamiento paliativo bien en Atención Primaria o compartido con Nefrología 32 .

\section{En el caso de pacientes diabéticos}

La derivación a Nefrología se hará teniendo en cuenta los criterios anteriores y además se remitirá a todo paciente con:
- Albuminuria: CAC (confirmada) > $300 \mathrm{mg} / \mathrm{g}$, a pesar de un adecuado tratamiento y control de la presión arterial (PA).

- Aumento de la albuminuria a pesar de un tratamiento adecuado.

- HTA refractaria (tres fármacos a dosis plenas y ausencia de control).

\section{Indicaciones de solicitud de ecografía desde Atención Primaria}

Bien para su seguimiento en Atención Primaria o para su derivación a Nefrología, se considera pertinente la solicitud de ecografía en el estudio diagnóstico de la ERC. Sus indicaciones son:

- ERC progresiva (disminución del FGe > $5 \mathrm{ml} /$ $\min / 1,73 \mathrm{~m}^{2}$ en un año).

- Hematuria macroscópica o albuminuria persistente.

- Sintomatología de obstrucción del tracto urinario.

- Edad > 15 años e historia familiar de riñones poliquísticos.

- Estadio 4 o 5. Valorar previamente comorbilidades asociadas.

- ERC con proteinuria.

- Infecciones urinarias de repetición con participación renal.

\section{MONITORIZACIÓN Y SEGUIMIENTO DE PACIENTES CON ENFERMEDAD RENAL CRÓNICA}

La frecuencia de la monitorización y las visitas de los pacientes con ERC se muestra en la tabla 6. En cualquier caso, es necesario individualizar estos criterios generales.

En cada revisión en Atención Primaria se recomienda:

- Controlar la PA y ajustar el tratamiento. Objetivo de $\mathrm{PA}<140 / 90 \mathrm{mmHg}$. En pacientes con proteinuria $(\mathrm{CAC}>300 \mathrm{mg} / \mathrm{g})$, se recomiendan cifras próximas a 130/80 mmHg. En pacientes de edad avanzada esta medida será objeto de una prudente y cuidada individualización $^{33}$. Evitar hipotensiones en pacientes de edad avanzada y enfermedad ateromatosa importante.

- Vigilar presencia de anemia: si ERC 3-5 y $\mathrm{Hb}<10,5 \mathrm{~g} / \mathrm{dl}$ (una vez descartada ferropenia: IST $>20 \%$ y ferritina $>100 \mathrm{ng} / \mathbf{m l}$ ), estimar remisión o adelantarla en Nefrología para valorar tratamiento con factores estimulantes de la eritropoyesis.

- Revisar la medicación, ajustando la dosis según el FG. En ERC 3-5, evitar la utilización de AINE, antidiabéticos orales de eliminación renal y contrastes yodados.

- Revisar los hábitos dietéticos, orientando al paciente sobre el tipo de dieta que seguir según el FG:

- ERC 1-3: solo se recomienda dieta hiposódica en caso de HTA. 
Tabla 6. Frecuencia de monitorización de visitas (número de visitas anuales)

\begin{tabular}{|c|c|c|c|c|}
\hline \multirow[b]{2}{*}{ Estadio ERC } & \multirow[b]{2}{*}{$\mathrm{FGe}\left(\mathrm{ml} / \mathrm{min} / 1,73 \mathrm{~m}^{2}\right)$} & \multicolumn{3}{|c|}{ Estadio de albuminuria } \\
\hline & & $\begin{array}{c}\mathrm{A} 1 \\
(<30 \mathrm{mg} / \mathrm{g})\end{array}$ & $\begin{array}{c}\mathrm{A} 2 \\
(30-300 \mathrm{mg} / \mathrm{g})\end{array}$ & $\begin{array}{c}\text { A3 (proteinuria) } \\
\text { (> } 300 \mathrm{mg} / \mathrm{g} \text { ) }\end{array}$ \\
\hline 1 & $>90$ & 1 si ERC & 1 & 2 \\
\hline 2 & $60-89$ & 1 si ERC & 1 & 2 \\
\hline $3 a$ & $45-59$ & 1 & 2 & 3 \\
\hline $3 b$ & $30-44$ & 2 & 3 & 3 \\
\hline 4 & $15-29$ & 3 & 3 & 40 más \\
\hline 5 & $<15$ & 4 o más & 40 más & 4 o más \\
\hline
\end{tabular}

Control por Nefrología.

Control por Atención Primaria u otras especialidades.

ERC: enfermedad renal crónica; FGe: filtrado glomerular estimado.

Nota: Los números de cada casilla son las visitas anuales.

- ERC 4-5: recomendaciones dietéticas sobre sodio, fósforo y potasio.

- Analítica en cada revisión a partir de ERC 3* (en negrita, lo mínimo aconsejable):

- Hemograma.

- Bioquímica sanguínea: glucosa, creatinina, urea, Na, K, Ca, P, albúmina y colesterol. FG estimado mediante MDRD o CKD-EPI.

- Bioquímica urinaria (muestra simple de orina de primera hora de la mañana): CAC.

- Sedimento de orina.

Se procurará compaginar las extracciones para no repetirlas. Se proporcionará al paciente un informe o, en su defecto, copia de los análisis. Si las revisiones en Nefrología se realizan mensualmente, no es necesario repetir los análisis en Atención Primaria.

En la tabla 7 se muestran los objetivos en la monitorización y seguimiento de pacientes con ERC según los estadios.

\section{PREVENCIÓN DE LA NEFROTOXICIDAD}

Una vez el paciente es diagnosticado de ERC, lo más importante es evitar que esta evolucione, y para ello tendremos que conocer que existen fármacos usados en la práctica diaria, y más concretamente en estos pacientes, que pueden provocar empeoramiento de esta entidad. Por otra parte, el uso de contrastes intravenosos de una manera indiscriminada y sin preparación previa puede provocar la nefropatía inducida por contraste. Por ello hay que evitar el uso de nefrotóxicos y minimizar el efecto y el uso de los contrastes intravenosos.
Evitar nefrotóxicos y tener precaución con fármacos que alteren la hemodinámica glomerular

1. Evitar la hiperpotasemia asociada a fármacos. Especial precaución debe tenerse con la asociación de un diurético ahorrador de potasio (espironolactona, amiloride, eplerenona) a otro fármaco que retenga potasio (IECA, ARA II, inhibidores directos de renina, AINE, betabloqueantes). En estos casos la monitorización frecuente del potasio sérico es obligada.

2. Evitar el uso de fármacos que producen depleción brusca de volumen y que pueden provocar daño tubular, sobre todo en situaciones de deshidratación, así como aquellos con efecto directo negativo sobre el túbulo (aminoglucósidos, tacrolimus, ciclosporina A).

3. Se debe hacer especial énfasis en evitar el uso innecesario de AINE, por el riesgo de deterioro de la función renal.

4. Ajustar los fármacos al FG, especialmente en el anciano y en el diabético. En estos pacientes deben utilizarse con precaución la metformina, (debe evitarse $\mathrm{su}$ inicio con $\mathrm{FGe}<30 \mathrm{ml} / \mathrm{min} / 1,73 \mathrm{~m}^{2}$ ) y $\operatorname{los}$ antidiabéticos orales de eliminación renal, los nuevos anticoagulantes, los antibióticos nefrotóxicos y algunas heparinas. Muchos fármacos producen nefrotoxicidad directa y su efecto deletéreo se agrava con la asociación de aquellos que interfieren la hemodinámica glomerular. Es muy importante evitarlos en situaciones de riesgo o ajustar su dosis según el FGe (aminoglucósidos, vancomicina, aciclovir, tenofovir, anfotericina, etc.). Se puede consultar el listado de fármacos para ajustar la dosis en la siguiente página web: http://nefrologiadigital.revistanefrologia.com/modules.php? name=libro\&op=viewCap\&idpublication $=1 \&$ idedition $=13$ \&idcapitulo $=109$. 
Tabla 7. Objetivos por especialidad en el seguimiento del paciente con enfermedad renal crónica

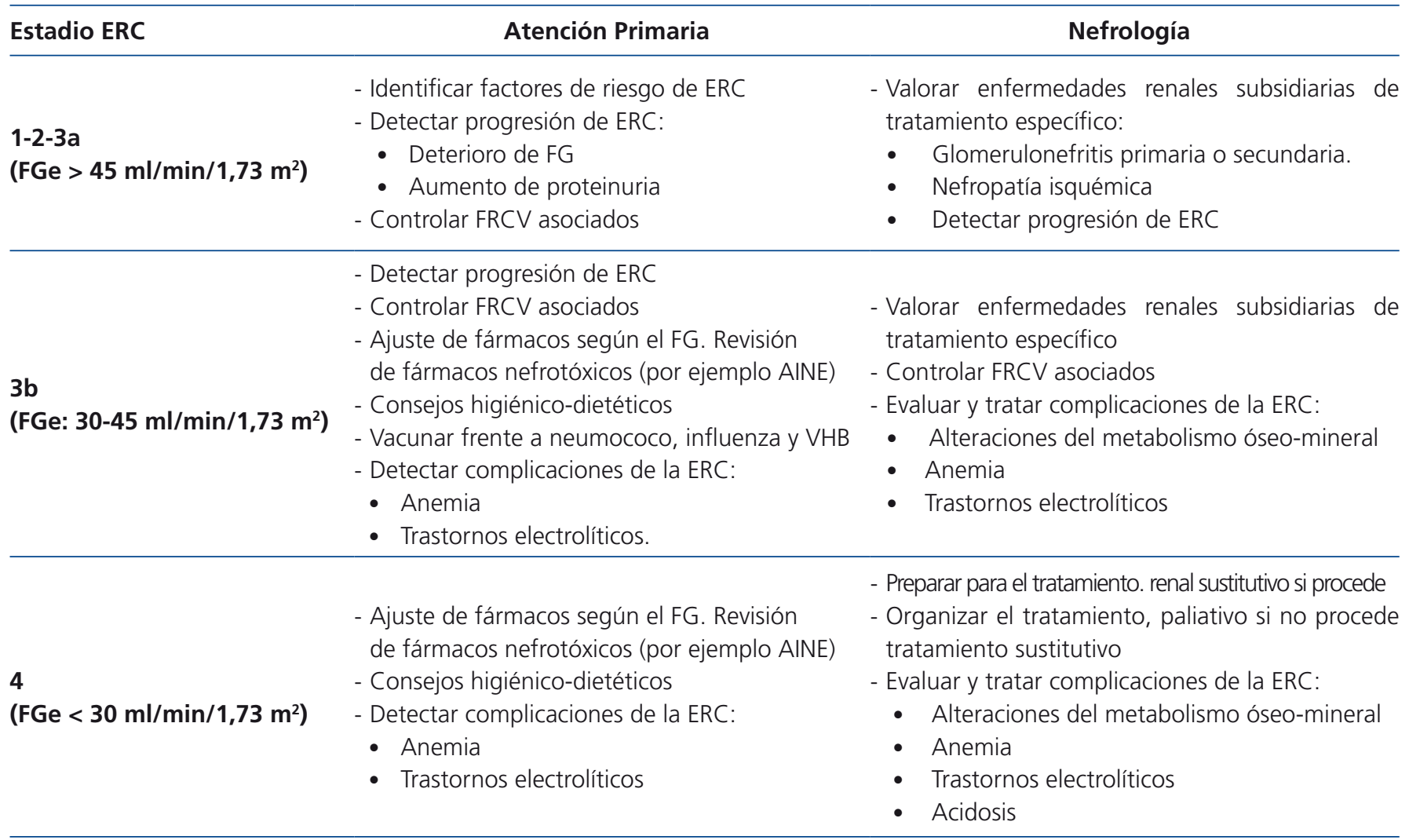

AINE: antiinflamatorios no esteroideos; ERC: enfermedad renal crónica; FG: filtrado glomerular; FGe: filtrado glomerular estimado; FRCV: factores de riesgo cardiovascular; VHB: virus de la hepatitis B.

\section{Minimizar el uso de contrastes intravenosos}

La nefropatía inducida por contraste se define como un deterioro de la función renal traducida en un aumento relativo de la creatinina de un $25 \%$ o un aumento absoluto de creatinina de $0,5 \mathrm{mg} / \mathrm{dl}$ respecto a la basal, que ocurre durante los tres primeros días tras la administración de contraste y que no se debe a ningún otro mecanismo. Se produce por toxicidad directa sobre las células tubulares ${ }^{34}$.

El aspecto más importante para prevenir la nefropatía por contraste es detectar a aquellos pacientes en riesgo de desarrollarla (edad avanzada, insuficiencia cardíaca, DM, insuficiencia renal previa, deshidratación, infarto agudo de miocardio, shock, volumen de contraste, anemia, hipotensión, uso de nefrotóxicos y altas dosis de diuréticos, cuidado con fármacos que alteren la hemodinámica glomerular y evitar hipotensión). El mejor tratamiento es la prevención, evitando situaciones de riesgo. Se recomienda la suspensión de diuréticos al menos 4-6 días antes de la administración de contraste, así como una correcta hidratación mediante fluidoterapia intravenosa e hidratación oral. Algunos fármacos pueden ser potencialmente tóxicos tras la administración de contraste, como por ejemplo la metformina. No obstante, en la actuali- dad, no existe evidencia suficiente para tener que suspender la metformina en pacientes con función renal previa normal a los que se les administra una cantidad «moderada» de contraste $^{35}$, aunque algunos autores lo sugieren.

\section{ACTITUDES, ESTILO DE VIDA Y TRATAMIENTO}

\section{Ejercicio físico}

Como norma general, se recomienda realizar 30-60 minutos de ejercicio moderado de 4 a 7 días por semana. Cuando la enfermedad renal está establecida, el ejercicio debe adaptarse a la capacidad física de cada paciente.

\section{Dieta}

Las recomendaciones dietéticas deben individualizarse para evitar sobrepeso u obesidad, pero también según la función renal del paciente y la existencia de otros factores de riesgo en los que esté indicada alguna restricción específica.

- ERC 1-3: solo se recomiendan dietas hiposódicas en caso de HTA y/o insuficiencia cardíaca. 


\section{documento de consenso}

- ERC 4-5: recomendaciones dietéticas sobre el sodio, el fósforo, el potasio y las proteínas.

Las necesidades energéticas son similares a las de la población general. La información disponible sugiere que la restricción proteica retrasa la progresión de la insuficiencia renal y debería empezar a aplicarse cuando el FG cae por debajo de los $30 \mathrm{ml} / \mathrm{min}$, salvo en casos de proteinuria por hiperfiltración, en cuyo caso debe instaurarse mucho antes, incluso con función renal normal. Debe ajustarse el contenido en proteínas a $0,8 \mathrm{~g} / \mathrm{kg} /$ día (al menos la mitad deben ser proteínas animales de alto valor biológico), pero con alto contenido calórico con base en grasas (mono y poliinsaturadas) e hidratos de carbono, si no existen intolerancia hidrocarbonada o dislipemia que exijan ajustes adicionales. La aportación adecuada de proteínas en pacientes con ERC es de $0,8 \mathrm{~g} / \mathrm{kg}$ de peso, el $50 \%$ de las cuales debe ser de alto valor biológico, es decir, de origen animal; el otro $50 \%$ debe completarse con las proteínas que contienen el resto de los alimentos incluidos en la dieta (de origen vegetal). Por otra parte, el uso de dietas de alto contenido proteico, así como de fármacos que produzcan reducción del peso, puede producir efectos adversos en la ERC ${ }^{36}$.

El consumo diario de sal ha de ser menor de $6 \mathrm{~g}$ (equivale a $2,4 \mathrm{~g}$ de sodio). En fases iniciales de la enfermedad renal, una restricción de sal más estricta se aplicará únicamente a los pacientes hipertensos. La dieta debe completarse con restricción de potasio y fósforo, y aporte de vitamina $\mathrm{D}$.

En pacientes con ERC en hemodiálisis (HD), la ingesta proteica puede aumentar hasta $1,2 \mathrm{~g} / \mathrm{kg}$ de peso para favorecer un adecuado balance proteico, evitar el desgaste calóricoenergético y lograr un adecuado estado nutricional.

\section{Alcohol}

No se considera perjudicial una ingesta moderada de alcohol, como en la población general, lo que supone unos 12 a $14 \mathrm{~g}$ de etanol (aproximadamente 300 cc de cerveza o 150 cc de vino). Pero hay que tener en cuenta no solo las calorías presentes en el alcohol, sino también la cantidad de líquido y el contenido en azúcar, potasio, fósforo y sodio, que debe limitarse en muchos pacientes según los factores de riesgo asociados y el grado de insuficiencia renal que presenten.

\section{OBJETIVOS ESPECÍFICOS DE TRATAMIENTO}

\section{Hipertensión arterial en la enfermedad renal} crónica: objetivos de tratamiento ${ }^{5,33,37}$

- En pacientes con ERC, el objetivo del tratamiento antihipertensivo es triple: reducción de la PA, reducción del riesgo de complicaciones cardiovasculares y enlentecimiento de la progresión de la ERC.
- En pacientes con ERC y un CAC $<30 \mathrm{mg} / \mathrm{g}$, se recomienda un objetivo de control de la $\mathrm{PA} \leq 140 / 90 \mathrm{mmHg}$. Si el CAC $\geq 30 \mathrm{mg} / \mathrm{g}$, se sugiere un objetivo más estricto, con $\mathrm{PA} \leq 130 / 80 \mathrm{mmHg}$, tanto en pacientes con DM como sin DM.

- Se aconsejan como fármacos de primera elección aquellos que bloqueen las acciones del sistema renina angiotensina, bien IECA o ARA.

- Se recomienda la utilización de combinaciones de fármacos antihipertensivos para alcanzar los objetivos de control. Dicha combinación debe incluir un diurético, tiazídico o de asa según la severidad de la ERC.

\section{Manejo de la hiperglucemia en la enfermedad renal crónica}

\section{Objetivos de manejo}

\section{¿Cómo valorar el control metabólico?}

La hemoglobina glucosilada $\mathrm{A}_{1 \mathrm{C}}\left(\mathrm{Hb}_{1 \mathrm{C}}\right)$ es el parámetro de referencia para valorar el control metabólico en el paciente con insuficiencia renal crónica (IRC), teniendo presentes los factores que limitan su utilidad como marcador de control glucémico, como las transfusiones y el tratamiento con eritropoyetina $^{38-43}$.

\section{Objetivos de control glucémico}

Las guías recomiendan que en los pacientes con DM de poco tiempo de evolución y sin disminución de la expectativa de vida el objetivo debe ser $\mathrm{HbA}_{1 \mathrm{C}}<7(<53 \mathrm{mmol} / \mathrm{mol})^{44}$.

Por el contrario, para los pacientes con DM de larga evolución con historia previa de mal control glucémico o con una condición que les disminuya las expectativas de vida, se debe individualizar el objetivo de control, evitando las estrategias terapéuticas que supongan un incremento inaceptable del riesgo de hipoglucemia.

No hay evidencias que indiquen cuál es el nivel óptimo de $\mathrm{HbA}_{1 \mathrm{C}}$ para los pacientes en diálisis.

Hay que tener en cuenta que el riesgo de presentar hipoglucemia severa en pacientes con insuficiencia renal en tratamiento intensivo es muy alto y está favorecido por la disminución en la ingesta, los cambios en los horarios de comidas y la presencia de neuropatía autonómica.

\section{Antidiabéticos orales ${ }^{44}$}

\section{Secretagogos}

Las sulfonilureas (SU) no son el fármaco de primera elección en la insuficiencia renal. 
Glibenclamida y glimepirida son metabolizadas en el hígado a metabolitos más débiles, pero de eliminación urinaria, por lo que su uso incluso en dosis bajas no es aconsejable en pacientes con IRC.

La glipizida es metabolizada a metabolitos inactivos; en consecuencia, sería la única SU que se puede administrar en IRC, pero su uso no está permitido con FG más bajos $(\mathrm{CCr}$ $<30 \mathrm{ml} / \mathrm{m})$.

La repaglinida tiene un metabolismo hepático con menos de un $10 \%$ de eliminación renal. A pesar de ello, cuando se instaure su tratamiento se debe iniciar con una dosis baja $0,5 \mathrm{mg}$.

\section{Metformina}

La metformina se elimina principalmente por la orina sin metabolizarse. En la ficha técnica se recomienda no utilizar con $\mathrm{FGe}<60 \mathrm{ml} / \mathrm{min}$, recomendación que no suele seguirse en la práctica clínica habitual. Con $\mathrm{FGe}<45 \mathrm{ml} /$ min se recomienda controlar la glucemia y la función renal frecuentemente y no administrarla con $\mathrm{FGe}<30 \mathrm{ml} / \mathrm{min}$.

\section{Inhibidores de $\alpha$-glucosidasa}

Tanto la acarbosa como el miglitol, como sus metabolitos, se acumulan en la insuficiencia renal, por lo que su uso no está recomendado ${ }^{45}$.

\section{Glitazonas}

Las glitazonas tienen un metabolismo hepático, excretándose menos del $2 \%$ por la orina. En consecuencia, no hay acumulación de metabolitos activos en la insuficiencia renal. Sin embargo, dado que su uso aumenta el riesgo de edema, insuficiencia cardíaca y osteoporosis, su uso está limitado en estos pacientes y contraindicado en aquellos en diálisis.

\section{Inhibidores de la dipeptidil peptidasa 4}

Con un FGe por encima de $50 \mathrm{ml} / \mathrm{min} / 1,73 \mathrm{~m}^{2}$ ninguna gliptina precisa ajuste. Sitagliptina, vildagliptina y saxagliptina precisan ajuste de dosis cuando el FG es menor de $50 \mathrm{ml} /$ minuto.

Sitagliptina se debe usar a dosis de $50 \mathrm{mg}$ y $25 \mathrm{mg}$ cuando el FG está entre 50 y $30 \mathrm{ml} /$ minuto y por debajo de $30 \mathrm{ml} /$ minuto, respectivamente.

Vildagliptina se debe emplear a dosis de $50 \mathrm{mg}$ por debajo de $50 \mathrm{ml} /$ minuto, incluida la ERC terminal que precise diálisis.

Saxagliptina se ha de emplear a dosis de $2,5 \mathrm{mg}$ en los pacientes con FG inferior a $50 \mathrm{ml} /$ minuto. Saxagliptina no tiene indicación de uso en pacientes con ERC terminal o diálisis.
Linagliptina no precisa ajuste de dosis en todos los estadios de la ERC.

\section{Análogos del péptido similar al glucagón tipo 1}

En el caso de liraglutida, está indicada solo en pacientes con $\mathrm{FGe}>60 \mathrm{ml} / \mathrm{min} / 1,73 \mathrm{~m}^{2}$. La experiencia con liraglutida y exenatida en este campo es muy escasa.

\section{Insulina}

Las necesidades de insulina son muy variables, por lo que la individualización del tratamiento es esencial. Como normas iniciales que deben adaptarse a cada paciente mediante la monitorización de glucosa, podemos señalar:

- $\quad \mathrm{CCr}>50$ : no se precisa ajuste de dosis.

- CCr 50-10: precisará una reducción del $75 \%$ de la dosis previa de insulina.

- $\mathrm{CCr}<$ 10: precisará una reducción del $50 \%$ de la dosis previa de insulina.

El régimen de insulina se adaptará al objetivo de control y puede ser una terapia convencional o un tratamiento intensivo, si bien conviene recordar que la pauta bolo-basal es la que presenta una tasa de hipoglucemias más baja.

\section{Objetivos y manejo de la dislipemia}

Uno de los factores que empeoran el daño renal y aceleran el deterioro funcional renal es la dislipemia, independientemente de su efecto promotor de la arterioesclerosis.

\section{Estratificación del riesgo}

De acuerdo con las últimas guías europeas, los sujetos con ERC deben considerarse de alto o muy alto riesgo cardiovascular, sin requerir aplicar escalas de riesgo. Así, la presencia de ERC con $\mathrm{FG}<60 \mathrm{ml} / \mathrm{min} / 1,73 \mathrm{~m}^{2}$ clasifica al sujeto como de muy alto riesgo cardiovascular ${ }^{46}$.

Debe realizarse sistemáticamente un despistaje de dislipemia. Aunque el colesterol LDL (lipoproteínas de baja densidad) es el principal predictor de riesgo, el colesterol no-HDL (lipoproteínas de alta densidad) puede serlo mejor, al igual que ocurre en los diabéticos o en el síndrome metabólico.

\section{Evidencias del beneficio de tratar la dislipemia en la enfermedad renal crónica}

Datos obtenidos de análisis post hoc apoyan la capacidad de las estatinas de reducir las complicaciones cardiovasculares en pacientes con estadios de ERC 2 y $3^{47,48}$. 
Los resultados en estadios 4 y 5 o en HD no son tan $\operatorname{claros}^{49,50}$. Sin embargo, en el estudio SHARP (Study of Heart and Renal Protection) se observó una reducción del $17 \%$ en los eventos cardiovasculares en los sujetos con ERC estadios 3, 4 y 5 tratados con simvastatina-ezetimiba frente a placebo ${ }^{51}$. Esta reducción no se observó en aquellos pacientes bajo tratamiento con diálisis.

\section{Recomendaciones sobre estilo de vida}

La dieta es el determinante principal de los niveles de colesterol. El consejo básico es, pues, el dietético. Se recomienda que el $30 \%$ o menos de las calorías totales procedan de alimentos grasos y que menos de un $10 \%$ sean grasas saturadas. En cuanto al consumo de colesterol, se aconseja que no se consuman más de $300 \mathrm{mg}$ diarios.

\section{Fármacos hipolipemiantes y enfermedad renal crónica}

\section{Estatinas}

En el caso de las estatinas no es necesario ajuste de dosis, salvo en ERC en estadios muy avanzados ${ }^{3-5}$ y solamente para aquellas de eliminación renal. Constituyen el tratamiento de elección. La ERC, al igual que la edad avanzada, el sexo femenino, un IMC bajo, la disfunción hepática, el consumo de alcohol, las enfermedades sistémicas y el hipotiroidismo, aumenta el riesgo de efectos secundarios, que por otro lado no son frecuentes.

Los fármacos de eliminación hepática serían de elección (fluvastatina, atorvastatina, pitavastatina y ezetimiba). Las estatinas metabolizadas vía CYP3A4 (atorvastatina, lovastatina, simvastatina) pueden aumentar los efectos secundarios por potenciar las interacciones farmacológicas cuando se administran con determinados fármacos inductores (fenitoína, fenobarbital, barbitúricos, rifampicina, dexametasona, ciclofosfamida, carbamacepina, omeprazol) o inhibidores.

En el caso de pacientes portadores de un trasplante renal, deben tenerse en cuenta determinadas interacciones, particularmente con ciclosporina y estatinas como atorvastatina, lovastatina y simvastatina, ya que pueden aumentar sus niveles y el riesgo de miopatía. Fluvastatina, pravastatina, pitavastatina y rosuvastatina tienen menos probabilidad de interaccionar. Aunque tacrolimus se metaboliza también por el CYP3A4, parece que posee menos riesgo de interaccionar con estatinas. En estos pacientes las estatinas deberán iniciarse a bajas dosis, titularse con cautela y vigilarse las interacciones.

\section{Fibratos}

La mayor parte de las guías recomiendan como fibrato de elección el gemfibrozilo y evitar el resto. El riesgo de miopa- tía se incrementa en más de cinco veces al asociarse a estatina y es mayor en caso de ERC. La asociación con estatinas puede provocar fracaso renal agudo por rabdomiolisis. En caso de requerirse la asociación, debe utilizarse fenofibrato y realizarse una monitorización estrecha. La dosis habitual de gemfibrocilo es de $600 \mathrm{mg} /$ día y puede emplearse en pacientes con FG entre 15-59 ml/min. Se desaconseja su uso si el FG es $<15 \mathrm{ml} / \mathrm{min}$. No obstante, dada la escasa evidencia del beneficio cardiovascular del tratamiento de la hipertrigliceridemia con fibratos y sus potenciales efectos secundarios, no se recomienda el tratamiento con fibratos en la ERC, especialmente cuando se asocien a estatinas.

\section{Ezetimiba}

Su eficacia junto con simvastatina ha sido demostrada en pacientes con ERC en el SHARP ${ }^{51}$. No se requiere ajuste de dosis en insuficiencia renal.

\section{Objetivos de control}

La ERC es un equivalente coronario; por lo tanto, los objetivos son los mismos que en el paciente con cardiopatía isquémica.

El objetivo terapéutico en el paciente con ERC $(\mathrm{FG}<60 \mathrm{ml} / \mathrm{m})$ es un colesterol $\mathrm{LDL}<70 \mathrm{mg} / \mathrm{dl}$ o una reducción del $50 \%$ si el objetivo previo no es alcanzable ${ }^{46}$. No obstante, recientemente las guías KDIGO para el tratamiento de la dislipemia en la ERC recomiendan la terapia con estatinas a todos los adultos $>50$ años, independientemente de los niveles de colesterol LDL. Basado en el estudio SHARP y en análisis post hoc de ensayos clínicos con estatinas frente a placebo que estudian a los pacientes con ERC, se establece una estrategia de «tratar el riesgo cardiovascular». De la misma forma, no recomiendan iniciar tratamiento con estatinas en pacientes con ERC estadio 5 en diálisis ${ }^{52}$.

Tras la elaboración del presente documento se han publicado las nuevas guías internacionales sobre el manejo de la dislipemia en la ERC (Kidney Int Suppl 2013;3(3):259-305), que sugieren no perseguir objetivos, sino una estrategia de «actuar».

\section{Tabaquismo}

El hábito de fumar representa uno de los factores directos involucrados en la progresión de la enfermedad renal y se han publicado datos de asociación entre este hábito y el deterioro de la función renal en población general (estudio MRFIT $^{53}$, estudio de Pinto-Sietsma et al. del estudio PREVEND $^{54}$, estudio de Halimi et al. ${ }^{55}$, estudio de Briganti et al. $\left.{ }^{56}\right)$ y en diabéticos ${ }^{57-60}$.

Por tanto, en todo paciente con ERC (igual que en la población general) debemos preguntar por el consumo de tabaco en 
todas las consultas que realicemos (tanto en primaria como en especializada). En los pacientes fumadores se realizará un consejo empático, pero firme y motivado, para dejar de fumar y se hará énfasis en los beneficios esperados individuales y en las posibles ayudas para conseguirlo (intervención mínima sistematizada, técnicas cognitivo-conductuales, tratamiento farmacológico, etc.).

En pacientes con enfermedad renal, parece segura la utilización de terapia de sustitución de nicotina (parches, chicles, caramelos) y su asociación con bupropión a dosis más bajas de la habitual $(150 \mathrm{mg} / 24 \mathrm{~h})$ en fases avanzadas de la enfermedad. El uso de vareniclina a dosis habituales parece seguro e, igualmente que con bupropión, se puede utilizar a mitad de dosis $(1 \mathrm{mg} / 24 \mathrm{~h})$ que en pacientes con función renal moderadamente disminuida y en población general.

\section{Obesidad}

Hay pocos ensayos clínicos especialmente diseñados, pero existen datos que apoyan que la reducción de peso y de la ingesta de grasas puede disminuir el riesgo de $\mathrm{ERC}^{61}$.

El tratamiento de la obesidad en pacientes con ERC debe ser no farmacológico y consistir en la realización de ejercicio físico y en una dieta hipocalórica siguiendo las recomendaciones del apartado correspondiente de esta guía.

El único fármaco autorizado en España para el tratamiento de la obesidad, orlistat, está indicado en personas con IMC superior a $30 \mathrm{~kg} / \mathrm{m}^{2}$. Presenta interacciones con numerosos fármacos y no ha sido estudiado en pacientes con ERC, por lo que no parece aconsejable su utilización en ellos. El uso de fármacos para reducir el apetito no está indicado en pacientes con ERC.

\section{Hiperuricemia}

La hiperuricemia se define como el aumento de los niveles de ácido úrico por encima de su límite de solubilidad en plasma. Esto ocurre en los varones con valores de ácido úrico superiores a $7 \mathrm{mg} / \mathrm{dl}$ y en mujeres, por efecto estrogénico, con valores superiores a $6 \mathrm{mg} / \mathrm{dl}$. La hiperuricemia puede ser asintomática u ocasionar enfermedades como nefrolitiasis úrica, nefropatía por ácido úrico, gota tofácea, artritis gotosa aguda e hiperuricemia asintomática. Se ha descrito un aumento del riesgo cardiovascular con valores de ácido úrico en el límite alto de la normalidad, por encima de $5,2 \mathrm{mg} / \mathrm{dl}^{62,63}$.

Se ha demostrado que el alopurinol y otros inhibidores de la xantina oxidasa tienen efectos sobre el aparato circulatorio que son independientes de la concentración de ácido úrico ${ }^{64-66}$.
Las guías clínicas no recomiendan el tratamiento de la hiperuricemia asintomática, puesto que solo se apoyaría en dos ensayos clínicos aleatorizados ${ }^{67,68}$. Recientemente se encuentra disponible otro fármaco, febuxostat, para el tratamiento de la hiperuricemia en pacientes con antecedentes de gota o artritis úrica. En aquellos con hiperuricemia sintomática e insuficiencia renal leve o moderada, la administración de febuxostat ha demostrado una eficacia superior y una seguridad similar al alopurinol, sin necesidad de ajustar dosis ${ }^{69}$.

La colchicina está indicada en el tratamiento del ataque agudo de gota. En pacientes con FG entre 30 y $50 \mathrm{ml} / \mathrm{min}$ debe reducirse la dosis. Por debajo de $30 \mathrm{ml} / \mathrm{min} / 1,73 \mathrm{~m}^{2}$, el uso de colchicina está contraindicado. En caso de crisis de gota aguda en estos pacientes con FGe reducido se puede administrar tetracosáctido (Nuvacthen depot $^{\circledR}$ ) intramuscular durante tres días o corticoides en dosis de 20-30 mg/día con reducción rápida hasta suspender en 5-7 días.

\section{Anemia}

La principal causa de anemia en la ERC es la producción inadecuada de eritropoyetina endógena, hormona que actúa sobre la diferenciación y maduración de los precursores de la serie roja. En pacientes con ERC, la anemia se define como la situación en la que la concentración de $\mathrm{Hb}$ en sangre se encuentra dos desviaciones estándar por debajo de la concentración media de $\mathrm{Hb}$ de la población general, corregida por edad y $\operatorname{sexo}^{70}$. El límite inferior de niveles de $\mathrm{Hb}$ a partir del cual se considera anemia en mujeres es de 11,5g/1 $1{ }^{71}$, según la S.E.N., y de 12 g/l, según la Organización Mundial de la Salud (OMS), la Kidney Disease Outcomes Quality Initiative (KDOQI) y la European Renal Best Practice (ERBP) ${ }^{72}$. El límite inferior de valores de $\mathrm{Hb}$ en varones de edad inferior a 70 años según la S.E.N., la KDOQI y la ERBP es de 13,5 g/l, y de $13 \mathrm{~g} / \mathrm{l}$ según la OMS. En el caso de varones de edad superior a 70 años, la S.E.N. y la OMS fijan los límites inferiores de $\mathrm{Hb}$ en $12 \mathrm{~g} / \mathrm{l}$, y la KDOQI y la ERBP en 13,5 g/l.

Diagnóstico de la anemia, evaluación y criterios para la administración de hierro y agentes estimulantes de la eritropoyesis

Características de la anemia en la enfermedad renal crónica La anemia asociada a la ERC habitualmente es normocítica y normocrómica en su origen y está relacionada con una disminución de la producción de eritropoyetina por las células peritubulares, baja respuesta de la médula ósea, producción aumentada de hepcidina y disminución de la disponibilidad de hierro para la eritropoyesis $^{73}$.

Cuándo iniciar el estudio de la anemia en la enfermedad renal crónica

- Cuando la cifra de Hb sea $<11$ g/dl en mujeres premenopáusicas y pacientes prepúberes. 
- Cuando la cifra de $\mathrm{Hb}$ sea $<12 \mathrm{~g} / \mathrm{dl}$ en varones adultos y mujeres posmenopáusicas.

Cómo estudiar la anemia de la enfermedad renal crónica, peticiones de laboratorio

- Hematocrito (Hto)-Hb.

- Hematimetría: volumen corpuscular medio (VCM), $\mathrm{Hb}$ corpuscular media (HCM), concentración de $\mathrm{Hb}$ corpuscular media (CHCM).

- Reticulocitos.

- Parámetros férricos: sideremia, ferritina, transferrina, IST.

- Descartar pérdidas sanguíneas intestinales (si anemia hipocroma microcítica o sospecha de sangrado digestivo).

- En pacientes con ERC-5 en HD, las muestras se sacan prediálisis inmediata.

\section{Objetivos de hemoglobina}

En el paciente con ERC deben buscarse objetivos de control de $\mathrm{Hb}$ entre 10 y $12 \mathrm{~g} / \mathrm{dl}$ en adultos, valorando síntomas y comorbilidad. Si en el paciente con ERC $3 \mathrm{~B}$ a 5 se comprueba $\mathrm{Hb}<10,5 \mathrm{~g} / \mathrm{dl}$, debe remitirse a Nefrología si no estaba en seguimiento o adelantar la revisión.

Metabolismo del hierro requerido previo al inicio de tratamiento con agentes estimulantes de la eritropoyesis Deben existir reservas suficientes para alcanzar y mantener el Hto/Hb objetivo:

- IST $\geq 20 \%$ y $<50 \%$.

Durante el tratamiento con agentes estimulantes de la eritropoyesis (AEE) se reitera el estudio del metabolismo férrico cada tres meses, si reciben Fe intravenoso.

En pacientes con AEE sin Fe intravenoso, el control debe ser mensual hasta estabilizar la $\mathrm{Hb}$, entre 10 y $12 \mathrm{~g} / \mathrm{dl}$.

En pacientes diabéticos, se recomienda no iniciar tratamiento con AEE hasta $\mathrm{Hb}<10 \mathrm{~g} / \mathrm{dl}$, si el paciente tiene antecedente de episodio de accidente vascular cerebral.

En pacientes no tratados con EPO, el objetivo ha de situarse en SatT $\geq 20 \%$ y Ferrit $\geq 100 \mathrm{ng} / \mathrm{ml}$. El control debe realizarse cada 3-6 meses.

La determinación debe ser 15 días después de la última dosis de $\mathrm{Fe}$ intravenoso.

Pauta de administración de hierro intravenoso

Se administra con el fin de prevenir el déficit y mantener las reservas férricas de modo que se alcance y mantenga el $\mathrm{Hto} / \mathrm{Hb}$ objetivo. La administración debe realizarse en el centro hospitalario. Con determinados protocolos conjuntos, algunos $\mathrm{Fe}$ intravenosos pueden suministrarse en el centro de salud bajo vigilancia médica.

\section{Pauta de administración de hierro por vía oral}

- Adultos: $200 \mathrm{mg} / \mathrm{día}$.

- Niños: 2-3 mg/kg/día.

En adultos prediálisis, en diálisis domiciliaria y DP que no logran adecuadas reservas férricas con Fe por vía oral, se administra una infusión $100 \mathrm{mg}$ de Fe dextrano o 500-1000 mg de $\mathrm{Fe}$ carboximaltosa intravenoso, repitiéndose las veces que sea necesario según parámetros férricos. Otra opción es hierro sacarosa intravenoso (máximo $200 \mathrm{mg} /$ dosis).

Se considera improbable que los pacientes en HD alcancen el objetivo con $\mathrm{Fe}$ oral, por lo que requerirán aporte de $\mathrm{Fe}$ intravenoso.

Vía de administración de agentes estimulantes de la eritropoyesis

Se indica la vía subcutánea en pacientes en HD, DP y diálisis domiciliaria, rotando el lugar de inyección.

La vía intraperitoneal sería posible administrando la dosis en abdomen vacío o con escasa cantidad de líquido peritoneal. Podrían requerirse dosis mayores por esta vía.

La vía intravenosa estaría indicada en caso de dosis elevadas (volumen) o equimosis recurrentes en el sitio de inyección.

Dosis de EPO y ajuste

Se pauta por los servicios de Nefrología.

Transfusiones en pacientes con enfermedad renal crónica

- En pacientes con síndrome funcional anémico.

- En pacientes resistentes a EPO con pérdidas sanguíneas crónicas.

Posibles efectos adversos secundarios al tratamiento con agentes estimulantes de la eritropoyesis

HTA, convulsiones, trombosis de fístula arteriovenosa, incremento de la viscosidad sanguínea. El tratamiento con AEE cuando se ha superado $\mathrm{Hb}>13 \mathrm{~g} / \mathrm{dl}$ se ha asociado con elevadas tasas de enfermedad cardiovascular, aunque sin incremento de la mortalidad ${ }^{74}$.

El control de la anemia en el paciente con ERC debe incluir programas de educación a pacientes que incluyan información sobre el problema de salud, soporte profesional y estilos de vida. 


\section{Detección de las alteraciones del metabolismo óseo y mineral}

Las alteraciones en el metabolismo del calcio y fósforo en la ERC se asocian con diversas complicaciones que van más allá de la simple afectación ósea e implican a otros sistemas, especialmente el cardiovascular (por ejemplo, calcificaciones). La manifestación clínica más temprana es el incremento de la hormona paratiroidea $(\mathrm{PTH})$, producida por déficit de vitamina D activa (calcitriol), retención de fosfato (con o sin hiperfosfatemia) y/o hiperfosfatemia franca.

\section{Objetivos de la detección y del tratamiento}

- Evitar la hiperfosfatemia.

- Mantener niveles normales de calcio y fósforo.

- Evitar la instauración y progresión del hiperparatiroidismo secundario.

Según las guías K-DOQI 2003, S.E.N. 2007 y S.E.N. $2011^{75-77}$, el objetivo terapéutico es variable en los diversos estadios de la ERC, pero se podría resumir diciendo que se debería intentar mantener dentro de límites normales el calcio, el fosfato y la PTH. En el estadio 4 se aconseja incluso mantener la PTH en valores ligeramente superiores a la normalidad. Las guías recomiendan también medir el calcidiol (25-OH-vitamina D) para diagnosticar el déficit o la insuficiencia de vitamina D. Idealmente estos niveles deberían ser mayores de $20-30 \mathrm{ng} / \mathrm{ml}$ (50-75 nmol/1).

Un pequeño grado de hiperparatiroidismo estable no es preocupante, pero el hiperparatiroidismo progresivo, con valores de PTH dos o tres veces superiores al valor de referencia, requieren una consulta al especialista de $\mathrm{Ne}$ frología. Niveles elevados de fosfato, superiores a $5 \mathrm{mg} /$ dl $(1,40 \mathrm{mmol} / \mathrm{l})$, son igualmente tributarios de consulta.

\section{Tratamiento}

Se realizará mediante dieta, captores del fósforo, vitamina D nativa o activa, y/o activación selectiva de los receptores de la vitamina D. En pacientes en diálisis se pueden utilizar calcimiméticos.

\section{Fármacos para un adecuado mantenimiento} del metabolismo mineral

\section{Captores del fósforo}

Se administran con las comidas. Destacan quelantes con calcio como el carbonato cálcico, el acetato cálcico o su asociación con magnesio. Entre los quelantes sin calcio ni aluminio, destacan el sevelamer y el carbonato de lantano. Los compuestos de aluminio son excelentes captores, pero no se recomiendan en períodos prolongados, dado que pueden inducir intoxicación alumínica en pacientes con ERC.

\section{Tratamiento del déficit de vitamina $D$}

- Colecalciferol (D3): vitamina D3 Belenguer ${ }^{\circledR}$ o Kern $^{\circledR}$ (colecalciferol; $800 \mathrm{UI}=12$ gotas, $50000 \mathrm{UI}=25 \mathrm{ml}$ ).

- Calcifediol (25-OH-vitamina D): Hidroferol ${ }^{\circledR} 0,266 \mathrm{mg}$ (calcifediol $16000 \mathrm{UI}$ ).

La dosificación de calcifediol en ampollas quincenales o mensuales tiene como objetivo normalizar los niveles de calcidiol $(25-\mathrm{OH}>20-30 \mathrm{ng} / \mathrm{ml})$ independientemente o no de disminuir la PTH. Debe manejarse con sumo cuidado y es obligatorio medir el calcio y el fósforo para su control, ya que en pacientes con ERC avanzada puede incrementarlos.

\section{Tratamiento del hiperparatiroidismo secundario}

- Calcitriol y análogos de la vitamina D: no necesitan hidroxilación renal para obtener la forma activa. Destacan el Rocaltrol $^{\circledR}$ (calcitriol; 1,25- $(\mathrm{OH})_{2}-\mathrm{D}_{3}$ ) y Etalpha $^{\circledR}$ (alfacalcidol; $\left.1 \alpha-(\mathrm{OH})-\mathrm{D}_{3}\right)$. El alfacalcidol requiere activación hepática.

- Activadores selectivos del receptor de la vitamina D: Zemplar $^{\circledR}$ (paricalcitol): ofrece menor tendencia a la hipercalcemia e hiperfosfatemia, y parece inducir menos calcificaciones vasculares.

- Calcimiméticos: Mimpara ${ }^{\circledR}$ (cinacalcet): indicado en el tratamiento del hiperparatiroidismo en diálisis y en hiperparatiroidismo primario.

\section{Detección y tratamiento de la acidosis}

Una situación de acidosis metabólica no compensada con bicarbonato venoso $<15 \mathrm{mmol} / 1$ requerirá tratamiento por vía endovenosa en ámbito hospitalario. Acidosis metabólicas leves (bicarbonato entre 15-20 mmol/l) pueden requerir administración de bicarbonato oral.

\section{Otras actitudes}

Preparación para el tratamiento renal sustitutivo y tiempo de iniciación

El inicio óptimo del tratamiento renal sustitutivo (TRS) es aquel que se realiza de forma planificada. La falta de previsión aumenta innecesariamente el uso de catéteres para HD, de los que se derivará un mayor morbilidad, infecciones e incremento en las hospitalizaciones. 


\section{documento de consenso}

Una remisión en tiempo adecuado del paciente al nefrólogo implica que el paciente recibirá una idónea información sobre las posibles técnicas de TRS: DP, HD, HD domiciliaria, así como la posibilidad de un trasplante renal anticipado o de donante vivo, si existiera. Esta remisión adecuada supone una disminución en las complicaciones, especialmente infecciosas y cardiovasculares, con un gran impacto en la supervivencia.

El TRS se plantea cuando el FG es $<15 \mathrm{ml} / \mathrm{min} / 1,73 \mathrm{~m}^{2} \mathrm{o}$ antes si aparecen signos o síntomas de uremia o dificultad en el control de la hidratación (hecho frecuente en el caso del paciente diabético), HTA de difícil control o empeoramiento del estado nutricional.

En general, se inicia diálisis cuando el FG está situado entre 8 y $10 \mathrm{ml} / \mathrm{min} / 1,73 \mathrm{~m}^{2}$ y es mandatorio con $\mathrm{FG}$ $<6 \mathrm{ml} / \mathrm{min} / 1,73 \mathrm{~m}^{2}$, incluso en ausencia de sintomatología urémica. En pacientes de alto riesgo, insistimos en que debe plantearse el inicio adelantado de diálisis, estableciéndolo de forma individualizada.

Hay que tener en cuenta que el paciente puede ser estudiado y preparado convenientemente para un posible trasplante renal de donante vivo (si existe esa posibilidad) sin que haya iniciado diálisis. Igualmente, puede ser estudiado y puesto en lista de espera para un posible trasplante renal de donante cadáver, si surge dicha posibilidad, antes del inicio de diálisis. Es lo que llamamos trasplante renal anticipado.

\section{Seguimiento}

Los pacientes con ERC 4-5 han de ser controlados preferentemente por el especialista nefrólogo, en estrecha colaboración con el médico de Atención Primaria y la enfermería.

La frecuencia de visitas debe establecerse cada tres meses en ERC 4 e incluso cada mes en ERC 5 prediálisis. Esta frecuencia podrá ser modificada según el criterio médico.

En cada visita es aconsejable aportar información detallada sobre analítica, modificaciones en el tratamiento, su justificación y, si procede, una valoración pronóstica.

\section{Funciones del médico de Atención Primaria en el abordaje y seguimiento de la enfermedad renal crónica condicionado por el estadio de la enfermedad}

- Seguimiento de los pacientes ancianos, con FG estable, que por motivos de edad, calidad de vida u otros, no sean tributarios de TRS y no reciban AEE y/o medicación para el hiperparatiroidismo secundario.

- Control de los factores de riesgo cardiovascular.
- Vigilancia de los factores de progresión de la ERC.

- Vigilancia de la nefrotoxicidad para evitar la iatrogenia en cualquier proceso.

- Debe prestarse especial atención a:

- Evitar siempre que sea posible el uso de AINE.

- Evitar la hiperpotasemia asociada al uso de fármacos.

- Evitar/adecuar el uso de antidiabéticos orales según el FGe.

- Evitar en lo posible el uso de contrastes yodados, ajustando cualquier fármaco al FGe del paciente.

- Participación en el cumplimiento terapéutico y derivación a Nefrología en caso de empeoramiento agudo de la función renal o aparición de complicaciones.

- Vacunación: virus de la hepatitis B, neumococo, gripe, otros.

- Colaboración en las actividades paliativas.

\section{Claves para el manejo del paciente en hemodiálisis/ diálisis peritoneal desde la Atención Primaria}

- Facilitación del proceso de adaptación a la enfermedad en función de la edad, la situación familiar, las condiciones de formación y laborales, la forma de aparición y desarrollo de la enfermedad, la confianza en el sistema sanitario, etc.

- Conocimiento por parte del médico de familia de las diferentes opciones e implementación de estas (periodicidad, lugar de realización, posibles complicaciones según alternativa elegida).

- Óptima relación y canal de comunicación con el Servicio de Nefrología de referencia.

\section{Claves para el manejo del paciente portador de un trasplante renal}

Las mismas que en el caso anterior, pero con interacción muy intensa con el nefrólogo, por los requerimientos especiales en relación con la inmunosupresión, las interacciones farmacológicas y las vacunaciones en estos casos.

\section{Claves para el seguimiento de la uremia terminal en domicilio. Tratamiento paliativo}

El manejo domiciliario de la uremia terminal tiene como objetivo facilitar el bienestar del paciente urémico que no puede ser sometido a diálisis, minimizando el impacto físico, familiar y asistencial de su condición, y optimizando los recursos de que dispone nuestro Sistema Nacional de Salud. Requiere una coordinación estrecha entre el Servicio de Nefrología y Atención Primaria. En aquellas áreas sanitarias donde existan equipos de soporte domiciliario, ya sean dependientes de Atención Primaria o de Atención 
Especializada, puede ser extraordinariamente útil su inclusión en el equipo terapéutico.

Se recomienda la individualización de la decisión con acuerdo del paciente, la familia y los profesionales. Es útil que la decisión se tome precozmente, ya que permite organizar el seguimiento antes de que se haya producido un deterioro especialmente significativo del paciente.

\section{Agradecimientos}

Agradecimientos a laboratorios Esteve y Abbvie por el apoyo logístico en el proyecto.

\section{Conflictos de interés}

Los autores declaran que no tienen conflictos de interés potenciales relacionados con los contenidos de este artículo.

\section{REFERENCIAS BIBLIOGRÁFICAS}

1. Otero A, de Francisco A, Gayoso P, García F; on behalf of the EPIRCE Study Group. Prevalence of chronic renal disease in Spain: results of the EPIRCE study. Nefrologia 2010;30(1):78-86.

2. De Francisco ALM. Sostenibilidad y equidad del tratamiento sustitutivo de la función renal en España. Nefrologia 2011;31:241-6.

3. Alcázar R, Egocheaga MI, Orte L, Lobos JM, González Parra E, Álvarez Guisasola F, et al. Documento de consenso sobre SEN-semFYC sobre la enfermedad renal crónica. Nefrologia 2008;28(3):273-82.

4. K/DOQI clinical practice guidelines for chronic kidney disease: evaluation, classification, and stratification. Am J Kidney Dis 2002;39(Suppl 1):S1-266.

5. National Collaborating Centre for Chronic Conditions. Chronic kidney disease: national clinical guideline for early identification and management in adults in primary and secondary care. London: Royal College of Physicians; 2008.

6. Improving Global Outcomes (KDIGO) CKD Work Group. KDIGO Clinical Practice Guideline for the Evaluation and Management of Chronic Kidney Disease. Kidney Int Suppl 2013;3:S6-308.

7. Levey AS, Stevens LA, Coresh J. Conceptual model of CKD: applications and implications. Am J Kidney Dis 2009;53(3 Suppl 3):S4-16.

8. Levey AS, Bosch JP, Lewis JB, Greene T, Rogers N, Roth D. A more accurate method to estimate glomerular filtration rate from serum creatinine: a new prediction equation. Modification of Diet in Renal Disease Study Group. Ann Intern Med 1999;130:461-70.

9. Levey AS, Stevens LA, Schmid CH, Zhang YL, Castro AF 3rd, Feldman $\mathrm{HI}$, et al. A new equation to estimate glomerular filtration rate. Ann Intern Med 2009;150:604-12.

10. Stevens LA, Schmid CH, Greene T, Zhang YL, Beck GJ, Froissart M, et al. Comparative performance of the CKD Epidemiology Collaboration (CKD-EPI) and the Modification of Diet in Renal Disease (MDRD) study equations for estimating GFR levels above $60 \mathrm{ml} / \mathrm{min} / 1.73 \mathrm{~m} 2$. Am J Kidney Dis 2010;56:486-95.
11. Peralta CA, Shlipak MG, Judd S, Cushman M, McClellan W, Zakai $N A$, et al. Detection of chronic kidney disease with creatinine, cystatin $C$, and urine albumin-to-creatinine ratio and association with progression to end-stage renal disease and mortality. JAMA 2011;305(15):1545-52.

12. Gràcia-Garcia S, Montañés-Bermúdez R, Morales-García LJ, Díezde Los Ríos MJ, Jiménez-García JÁ, Macías-Blanco C, et al. Current use of equations for estimating glomerular filtration rate in Spanish laboratories. Nefrologia 2012;32(4):508-16.

13. Stevens LA, Coresh J, Greene T, Levey AS. Assessing kidney function-measured and estimated glomerular filtration rate. N Engl J Med 2006;354:2473-83.

14. Cockroft DW, Gault MH. Prediction of creatinine clearance from serum creatinine. Nephron 1976;16:31-41.

15. Jones GR. Estimating renal function for drug dosing decisions. Clin Biochem Rev 2011;32:81-8.

16. Stevens LA, Nolin TD, Richardson MM, Feldman HI, Lewis JB, Rodby $R$, et al. Comparison of drug dosing recommendations based on measured GFR and kidney function estimating equations. Am J Kidney Dis 2009;54:33-42.

17. National Collaborating Centre for Chronic Conditions. Chronic kidney disease: national clinical guideline for early identification and management in adults in primary and secondary care. London: Royal College of Physicians; 2008.

18. Levey A, Coresh J. Chronic kidney disease. Lancet 2011;14;379:165-8.

19. Remuzzi G, Benigni A, Remuzzi A. Mechanisms of progression and regression of renal lesions of chronic nephropathies and diabetes. J Clin Invest 2006;116:288-96.

20. Hallan SI, Ritz E, Lydersen S, Romundstad S, Kvenild K, Orth SR. Combining GFR and albuminuria to classify CKD improves prediction of ESRD. J Am Soc Nephrol 2009;20:1069-77.

21. Klahr S, Levey AS, Beck GJ, Caggiula AW, Hunsicker L, Kusek $J W$, et al. The effects of dietary protein restriction an blood pressure control on the progression of chronic renal disease. Modification of Diet in Renal Disease Study Group. N Engl J Med 1994;330:877-84.

22. Hallan SI, Coresh J, Astor BC, Asberg A, Powe NR, Romundstad $S$, et al. International comparison of the relationship of chronic kidney disease prevalence and ESRD risk. J Am Soc Nephrol 2006; 17:2275-84.

23. Patel A, MacMahon S, Chalmers J, Neal B, Billot L, Woodward $M$, et al.; ADVANCE Collaborative Group. Intensive blood glucose control and vascular outcomes in patients with type 2 diabetes. N Engl J Med 2008;358:2560-72.

24. Elsayed EF, Tighiouart H, Griffith J, Kurth T, Levey AS, Salem D, et al. Cardiovascular disease and subsequent kidney disease. Arch Intern Med 2007;167:1130-6.

25. Orth SR, Schroeder T, Ritz E, Ferrari P. Effects of smoking on renal function in patients with type 1 and type 2 diabetes mellitus. Nephrol Dial Transplant 2005;20:2414-9.

26. Duran-Perez EG, Almeda-Valdes P, Cuevas-Ramos D, CamposBarrera E, Muñoz-Hernandez L, Gomez-Perez FJ. Treatment of metabolic syndrome slows progression of diabetic nephropathy. Metab Syndr Relat Disord 2011;9:483-9.

27. Roderick PJ, Raleigh VS, Hallam L, Mallick NP. The need and de- 
mand for renal replacement therapy in ethnic minorities in England. J Epidemiol Community Health 1996;50(3):334-9.

28. Morlans M, Laporte JR, Vidal X, Cabeza D, Stolley PD. End-stage renal disease and non-narcotic analgesics: A case-control study. $\mathrm{Br}$ J Clin Pharmacol 1990;30(5):717-23.

29. Navaneethan SD, Aloudat S, Singh S. A systematic review of patient and health system characteristics associated with late referral in chronic kidney disease. BMC Nephrology 2008;9:3.

30. Van Pottelbergh G, Bartholomeeusen S, Bunix F, Degryse J. The evolution of renal function and the incidence of end-stage renal disease in patients aged $\geq 50$ years. Nephrol Dial Transplant 2012;27(6):2297-303.

31. Campbell KH, Smith SG, Hemmerich J, Stankus N, Fox C, Mold $J W$, et al. Patient and provider determinants of nephrology referral in older adults with severe chronic kidney disease: a survey of provider decision-making. BMC Nephrology 2011;12:47.

32. Tejedor A, de las Cuevas X. Cuidado paliativo en el paciente con enfermedad renal crónica avanzado (estadio 5) no susceptible de tratamiento dialítico. Nefrologia 2008;28 Suppl 3:129-36.

33. KDIGO Clinical Practice Guidelines for the management of blood pressure in chronic kidney disease. Kidney Int 2012;2(Suppl):340-414.

34. Ueda J, Nygren A, Hansell P, Erikson U. Influence of contrast media on single nephron glomerular filtration rate in rat kidney. A comparison between diatrizoate, iohexol, ioxaglate, and iotrolan. Acta Radiol 1992;33:596-9.

35. Goergen SK. Systematic review of current guidelines, and their evidence base, on risk of lactic acidosis after administration of contrast medium for patients receiving metformin. Radiology 2010;254:261-9

36. Navaneethan SD, Kirwan JP, Arrigain S, Schreiber MJ, Sehgal R, Schold JD. Overweight, obesity and intentional weight loss in chronic kidney disease: NHANES 1999-2006. Int J Obes (Lond) 2012;36(12):1585-90.

37. Upadhyay A, Earley A, Haynes SM, Uhlig K. Systematic review: blood pressure target in chronic kidney disease and proteinura as an effect modifier. Ann Intern Med 2011;154(8):541-8.

38. Ansari A, Thomas S, Goldsmith D. Assessing glycemic control in patients with diabetes and end-stage renal failure. Am J Kidney Dis 2003;41:523-31.

39. de Boer MJ, Miedema K, Casparie AF. Glycosylated haemoglobin in renal failure. Diabetologia 1980;18:437-40.

40. Scott MG, Hoffman JW, Meltzer VN, Siegfried BA, Chan KM. Effects of azotemia on results of the boronate-agarose affinity method and in exchange methods for glycated hemoglobin. Clin Chem 1984;30:896-8.

41. Wettre S, Lundberg M. Kinetics of glycosylated hemoglobin in uremia determined on ion-exchange and affinity chromatography. Diabetes Res 1986;3:107-10.

42. Paisey R, Banks R, Holton R, Young K, Hopton M, White D, et al. Glycosylated haemoglobin in uraemia. Diabet Med 1986;3:445-8.

43. Joy MS, Cefalu WT, Hogan SL, Nachman PH. Long-term glycemic control measurements in diabetic patients receiving hemodialysis. Am J Kidney Dis 2002;39:297-307.

44. Gómez-Huelgas R, Martínez-Castelao A, Artola S, Górriz JL, Menéndez E, en nombre del Grupo de Trabajo para el Docu- mento de Consenso sobre el tratamiento de la diabetes tipo 2 en el paciente con enfermedad renal crónica. Documento de Consenso sobre el tratamiento de la diabetes tipo 2 en el paciente con enfermedad renal crónica. Nefrologia 2014;34(1):3445.

45. Snyder RW, Berns JS. Use of insulin and oral hypoglycemic medications in patients with diabetes mellitus and advanced kidney disease. Semin Dial 2004;17:365-9.

46. Reiner Z, Catapano AL, De Backer G, Graham I, Taskinen MR, Wiklund $O$, et al. ESC/EAS Guidelines for the management of dyslipidaemias: the Task Force for the management of dyslipidaemias of the European Society of Cardiology (ESC) and the European Atherosclerosis Society (EAS). Eur Heart J 2011;32:1769-818.

47. Tonelli M, Keech A, Shepherd J, Sacks F, Tonkin A, Packard C, et al. Effect of pravastatin in people with diabetes and chronic kidney disease. J Am Soc Nephrol 2005;16:3748-54.

48. Collins R, Armitage J, Parish S, Sleigh P, Peto R; Heart Protection Study Collaborative Group. MRC/BHF Heart Protection Study of cholesterol-lowering with simvastatin in 5963 people with diabetes: a randomised placebo-controlled trial. Lancet 2003;361:2005-16.

49. Wanner C, Krane V, März W, Olschewski M, Mann JF, Ruf G, et al.; German Diabetes and Dialysis Study Investigators. Atorvastatin in patients with type 2 diabetes mellitus undergoing hemodialysis. N Engl J Med 2005;353:238-48.

50. Fellström BC, Jardine AG, Schmieder RE, Holdaas H, Bannister K, Beutler J, et al.; for the AURORA Study Group. Rosuvastatin and cardiovascular events in patients undergoing hemodialysis. N Engl J Med 2009;360:1395-407.

51. SHARP Collaborative Group. Study of Heart and Renal Protection (SHARP): randomized trial to assess the effects of lowering low-density lipoprotein cholesterol among 9,438 patients with chronic kidney disease. Am Heart J 2010;160:785-94.

52. KDIGO Clinical Practice Guideline for Lipid Management in Chronic Kidney Disease. Kidney Int 2013 (Suppl);3:259-305.

53. Klag MJ, Whelton PK, Randall BL, Neaton JD, Brancati FL, Ford CE, et al. Blood pressure and end-stage renal disease in men. $\mathrm{N}$ Engl J Med 1996;334:13-8.

54. Pinto-Sietsma SJ, Mulder J, Janssen WM, Hillege HL, de Zeeuw D, de Jong PE. Smoking is related to albuminuria and abnormal renal function in nondiabetic persons. Ann Intern Med 2000;133:585-91.

55. Halimi JM, Giraudeau B, Vol S, Cacès E, Nivet H, Lebranchu Y, et al. Effects of current smoking and smoking discontinuation on renal function and proteinuria in the general population. Kidney Int 2000;58:1285-92.

56. Briganti EM, Branley P, Chadban SJ, Shaw JE, McNeil JJ, Welborn TA, et al. Smoking is associated with renal impairment and proteinuria in the normal population: The AusDiab kidney study. Australian Diabetes, Obesity and Lifestyle Study. Am J Kidney Dis 2002;40:704-12.

57. Verhave JC, Hillege HL, Burgerhof JG, Gansevoort RT, de Zeeuw D, de Jong PE; PREVEND Study Group. The association between atherosclerotic risk factors and renal function in the general population. Kidney Int 2005;67:1967-73.

58. de Boer IH, Sibley SD, Kestenbaum B, Sampson JN, Young B, Cleary PA, et al.; Diabetes Control and Complications Trial/Epidemiology of Diabetes Interventions and Complications Study Research Group. Central obesity, incident microalbuminuria, and change in creatinine 
clearance in the epidemiology of diabetes interventions and complications study. J Am Soc Nephrol 2007;18:235-43.

59. Rossing K, Christensen PK, Hovind P, Tarnow L, Rossing P, Parving $\mathrm{HH}$. Progression of nephropathy in type 2 diabetic patients. Kidney Int 2004;66:1596-605.

60. Scott LJ, Warram JH, Hanna LS, Laffel LM, Ryan L, Krolewski AS. A nonlinear effect of hyperglycemia and current cigarette smoking are major determinants of the onset of microalbuminuria in type 1 diabetes. Diabetes 2001;50:2842-9.

61. Navaneethan SD, Yehnert H, Moustarah F, Schreiber MJ, Schauer PR, Beddhu S. Weight loss interventions in chronic kidney disease: a systematic review and meta-analysis. Clin J Am Soc Nephrol 2009;4:1565-74.

62. Feig DI, Kang DH, Johnson RJ. Uric acid and cardiovascular risk. N Engl J Med 2008;359(17):1811-21.

63. Niskanen LK, Laaksonen DE, Nyyssönen K. Uric acid level as a risk factor for cardiovascular and all-cause mortality in middle-aged men: a prospective cohort study. Arch Intern Med 2004;164:1546-51.

64. George J, Carr E, Davies J, Belch JJF, Struthers A. High-dose allopurinol improves endothelial function by profoundly reducing vascular oxidative stress and not by lowering uric acid. Circulation 2006;114(23):2508-16.

65. Hare JM, Mangal B, Brown J, Fisher C, Freudenberger R, Colucci WS, et al. Impact of oxypurinol in patients with symptomatic heart failure. Results of the OPT-CHF Study. J Am Coll Cardiol 2008;51(24):2301-9.

66. Noman A, Ang DS, Ogston S, Lang CC, Struthers AD. Effect of high-dose allopurinol on exercise in patients with chronic stable angina: a randomised, placebo controlled crossover trial. Lancet 2010;375(9732):2161-7.

67. Feig DI, Soletsky B, Johnson RJ. Effect of allopurinol on blood pressure of adolescents with newly diagnosed essential hypertension. A randomized trial. JAMA 2008;300:924-32.

68. Goicoechea M, De Vinuesa SG, Verdalles U, Ruiz Caro C, Ampuero J, Rincón A, et al. Effect of allopurinol in chronic kidney disease progression and cardiovascular risk. Clin J Am Soc Nephrol 2010;5(8):1388-93.

69. Becker MA, Schumacher HR, Espinoza LR, Wells AF, MacDonald $P$, Lloyd $E$, et al. The urate-lowering efficacy and safety of febuxostat. Arthritis Res Ther 2010;12(2):R63.

70. Locatelli F, Aljama P, Bárány P, Canaud B, Carrera F, Eckardt KU, et al.; European Best Practice Guidelines Working Group. Revised European best practice guidelines for the management of anaemia in patients with chronic renal failure. Nephrol Dial Transplant 2004;19 Suppl 2:ii1-47.

71. López Gómez JM. Manejo de la anemia en la enfermedad renal crónica. Nefrologia 2008;28 Suppl 3:63-6.

72. Fiona M, Klarenbach S, Tonelli M, Johnson JA, Manns BJ. The impact of selecting high haemoglobin target level on health-related quality of life for patients with chronic kidney disease. Arch Intern Med 2009;169(12):1104-12.

73. Levey AS, Coresh J. Chronic kidney disease. Lancet 2012;379(9811):165-80.

74. Solomon SD, Uno H, Lewis EF, Eckardt KU, Lin J, Burdmann EA, et al.; for the Trial to Reduce Cardiovascular Events with Aranesp Therapy (TREAT) Investigators. Erythropoietic response and outcomes in kidney disease and type 2 diabetes. N Engl J Med 2010;363:1146-55.

75. National Kidney Foundation. K/DOQI clinical practice guidelines for bone metabolism and disease in chronic kidney disease. Am J Kidney Dis 2003;42(Suppl 3):S1-201.

76. Torregrosa JV, Cannata-Andia J, Bover J, Caravaca F, Lorenzo V, Martín de Francisco AL, et al.; SEN Guidelines. Recommendations of the Spanish Society of Nephrology for managing bone-mineral metabolic alterations in chronic renal disease patients. Nefrologia 2008;28 Suppl 1:1-22.

77. Torregrosa JV, Bover J, Cannata-Andía J, Lorenzo V, de Francisco AL, Martínez I, et al. Spanish Society of Nephrology recommendations for controlling mineral and bone disorder in chronic kidney disease patients (S.E.N.-M.B.D.). Nefrologia 2011;31 Suppl 1:3-32. 